\title{
Quotient of Bergman kernels on punctured Riemann surfaces
}

\author{
Hugues Auvray ${ }^{1} \cdot$ Xiaonan $\mathrm{Ma}^{2} \cdot$ George Marinescu ${ }^{3}$
}

Received: 25 July 2021 / Accepted: 13 January 2022 / Published online: 18 February 2022

(c) The Author(s) 2022

\begin{abstract}
In this paper we consider a punctured Riemann surface endowed with a Hermitian metric that equals the Poincaré metric near the punctures, and a holomorphic line bundle that polarizes the metric. We introduce a new method to compare the Bergman kernels of high tensor powers of the line bundle and of the Poincare model near the singularity and show that their quotient tends to one uniformly on a neighborhood of the singularity up to arbitrary negative powers of the tensor power.
\end{abstract}

\section{Contents}

1 Introduction . . . . . . . . . . . . . . . . . . . . . . . . . 2340

$2 C^{0}$-estimate for the quotient of Bergman kernels . . . . . . . . . . . . . . . . . . . . 2343

2.1 Proof of Theorem $1.2 \ldots \ldots \ldots \ldots \ldots \ldots$

2.2 Proof of Lemma $2.1 \ldots \ldots \ldots$. . . . . . . . . . . . . . . . . . . . . . . . . 2348

$3 C^{k}$-estimate of the quotient of Bergman kernels f . . . . . . . . . . . . . . . . . 2351

3.1 A refined integral estimate . . . . . . . . . . . . . . . . . . . 2351

3.2 Proof of Theorem $1.3 \ldots \ldots \ldots \ldots \ldots \ldots \ldots \ldots$

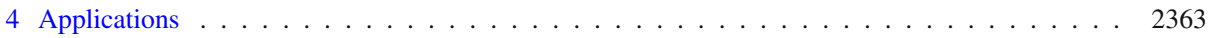

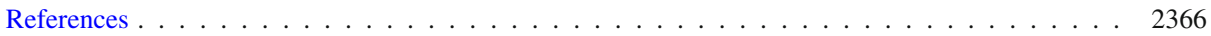

Hugues Auvray is partially supported by ANR contracts ANR-14-CE25-0010 and ANR-15-CE40-0003. Xiaonan Ma is partially supported by NSFC no. 11829102 and funded through the Institutional Strategy of the University of Cologne within the German Excellence Initiative. George Marinescu is partially supported by CRC TRR 191.

$\bowtie$ George Marinescu

gmarines@math.uni-koeln.de

Hugues Auvray

hugues.auvray@math.u-psud.fr

Xiaonan Ma

xiaonan.ma@imj-prg.fr

1 Laboratoire de Mathématiques d'Orsay, Département de Mathématiques, Université Paris-Sud, CNRS, Université Paris-Saclay, Bâtiment 307, 91405 Orsay, France

2 Université de Paris and Sorbonne Université, CNRS, IMJ-PRG, 75013 Paris, France

3 Department of Mathematics and Computer Science, Universität zu Köln, Weyertal 86-90, 50931

Cologne, Germany 


\section{Introduction}

In this paper we study the asymptotics of Bergman kernels of high tensor powers of a singular Hermitian line bundle over a Riemann surface under the assumption that the curvature has singularities of Poincaré type at a finite set. We show namely that the quotient of these Bergman kernels and of the Bergman kernel of the Poincaré model near the singularity tends to one up to arbitrary negative powers of the tensor power. In our previous paper [5] (see also [4]) we obtained a weighted estimate in the $C^{m}$-norm near the punctures for the difference of the global Bergman kernel and of the Bergman kernel of the Poincaré model near the singularity, uniformly in the tensor powers of the given bundle. Our method is inspired by the analytic localization technique of Bismut-Lebeau [7].

There exists a well-known expansion of the Bergman kernel on general compact manifolds $[8,11,14,20,22,23,28,29]$ with important applications to the existence and uniqueness of constant scalar curvature Kähler metrics $[17,28]$ as part of the Tian-Yau-Donaldson's program. Coming to our context, a central problem is the relation between the existence of special complete/singular metrics and the stability of the pair $(X, D)$ where $D$ is a smooth divisor of a compact Kähler manifold $X$; see e.g. the suggestions of [27, §3.1.2] for the case of " asymptotically hyperbolic Kähler metrics ", which naturally generalize to higher dimensions the complete metrics $\omega_{\Sigma}$ studied here. Moreover, the technique developed here can be extended to the higher dimensional situation in the case of Poincaré type Kähler metrics with reasonably fine asymptotics on complement of divisors, see the construction of $[2, \S 1.1]$ and [3, Theorem 4].

The Bergman kernel function of a singular polarization is of particular interest in arithmetic situations $[6,9,10]$. In [5] we applied the precise asymptotics of the Bergman kernel near the punctures in order to obtain optimal uniform estimates for the supremum of the Bergman kernel, relevant in arithmetic geometry $[1,18,21]$. There are also applications to " partial Bergman kernels", see [13].

We place ourselves in the setting of [5] which we describe now. Let $\bar{\Sigma}$ be a compact Riemann surface and let $D=\left\{a_{1}, \ldots, a_{N}\right\} \subset \bar{\Sigma}$ be a finite set. We consider the punctured Riemann surface $\Sigma=\bar{\Sigma} \backslash D$ and a Hermitian form $\omega_{\Sigma}$ on $\Sigma$. Let $L$ be a holomorphic line bundle on $\bar{\Sigma}$, and let $h$ be a singular Hermitian metric on $L$ such that:

( $\alpha) h$ is smooth over $\Sigma$, and for all $j=1, \ldots, N$, there is a trivialization of $L$ in the complex neighborhood $\overline{V_{j}}$ of $a_{j}$ in $\bar{\Sigma}$, with associated coordinate $z_{j}$ such that $|1|_{h}^{2}\left(z_{j}\right)=$ $\left|\log \left(\left|z_{j}\right|^{2}\right)\right|$.

( $\beta$ ) There exists $\varepsilon>0$ such that the (smooth) curvature $R^{L}$ of $h$ satisfies $i R^{L} \geq \varepsilon \omega_{\Sigma}$ over $\Sigma$ and moreover, $i R^{L}=\omega_{\Sigma}$ on $V_{j}:=\overline{V_{j}} \backslash\left\{a_{j}\right\}$; in particular, $\omega_{\Sigma}=\omega_{\mathbb{D}^{*}}$ in the local coordinate $z_{j}$ on $V_{j}$ and $\left(\Sigma, \omega_{\Sigma}\right)$ is complete.

Here $\omega_{\mathbb{D}^{*}}$ denotes the Poincaré metric on the punctured unit disc $\mathbb{D}^{*}$, normalized as follows:

$$
\omega_{\mathbb{D}^{*}}:=\frac{i d z \wedge d \bar{z}}{|z|^{2} \log ^{2}\left(|z|^{2}\right)} .
$$

For $p \geq 1$, let $h^{p}:=h^{\otimes p}$ be the metric induced by $h$ on $\left.L^{p}\right|_{\Sigma}$, where $L^{p}:=L^{\otimes p}$. We denote by $H_{(2)}^{0}\left(\Sigma, L^{p}\right)$ the space of $\boldsymbol{L}^{2}$-holomorphic sections of $L^{p}$ relative to the metrics $h^{p}$ and $\omega_{\Sigma}$,

$$
H_{(2)}^{0}\left(\Sigma, L^{p}\right)=\left\{S \in H^{0}\left(\Sigma, L^{p}\right):\|S\|_{\mathbf{L}^{2}}^{2}:=\int_{\Sigma}|S|_{h^{p}}^{2} \omega_{\Sigma}<+\infty\right\}
$$


endowed with the obvious inner product. The sections from $H_{(2)}^{0}\left(\Sigma, L^{p}\right)$ extend to holomorphic sections of $L^{p}$ over $\bar{\Sigma}$, i.e., (see [22,(6.2.17)])

$$
H_{(2)}^{0}\left(\Sigma, L^{p}\right) \subset H^{0}\left(\bar{\Sigma}, L^{p}\right) .
$$

In particular, the dimension $d_{p}$ of $H_{(2)}^{0}\left(\Sigma, L^{p}\right)$ is finite.

We denote by $B_{p}(\cdot, \cdot)$ and by $B_{p}(\cdot)$ the (Schwartz-)Bergman kernel and the Bergman kernel function of the orthogonal projection $B_{p}$ from the space of $\boldsymbol{L}^{2}$-sections of $L^{p}$ over $\Sigma$ onto $H_{(2)}^{0}\left(\Sigma, L^{p}\right)$. They are defined as follows: if $\left\{S_{\ell}^{p}\right\}_{\ell=1}^{d_{p}}$ is an orthonormal basis of $H_{(2)}^{0}\left(\Sigma, L^{p}\right)$, then

$$
B_{p}(x, y):=\sum_{\ell=1}^{d_{p}} S_{\ell}^{p}(x) \otimes\left(S_{\ell}^{p}(y)\right)^{*} \quad \text { and } \quad B_{p}(x):=\sum_{\ell=1}^{d_{p}}\left|S_{\ell}^{p}(x)\right|_{h^{p}}^{2} .
$$

Note that these are independent of the choice of basis (see [22,(6.1.10)] or [12,Lemma 3.1]). Similarly, let $B_{p}^{\mathbb{D}^{*}}(x, y)$ and $B_{p}^{\mathbb{D}^{*}}(x)$ be the Bergman kernel and Bergman kernel function of $\left(\mathbb{D}^{*}, \omega_{\mathbb{D}^{*}}, \mathbb{C},\left|\log \left(|z|^{2}\right)\right|^{p} h_{0}\right)$ with $h_{0}$ the flat Hermitian metric on the trivial line bundle $\mathbb{C}$.

Note that for $k \in \mathbb{N}$, the $C^{k}$-norm at $x \in \Sigma$ is defined for $\sigma \in C^{\infty}\left(\Sigma, L^{p}\right)$ as

$$
|\sigma|_{C^{k}\left(h^{p}\right)}(x)=\left(|\sigma|_{h^{p}}+\left|\nabla^{p, \Sigma} \sigma\right|_{h^{p}, \omega_{\Sigma}}+\cdots+\left|\left(\nabla^{p, \Sigma}\right)^{k} \sigma\right|_{h^{p}, \omega_{\Sigma}}\right)(x),
$$

where $\nabla^{p, \Sigma}$ is the connection on $(T \Sigma)^{\otimes \ell} \otimes L^{p}$ induced by the Levi-Civita connection on $\left(T \Sigma, \omega_{\Sigma}\right)$ and the Chern connection on $\left(L^{p}, h^{p}\right)$, and the pointwise norm $|\cdot|_{h^{p}, \omega_{\Sigma}}$ is induced by $\omega_{\Sigma}$ and $h^{p}$. In the same way we define the $C^{k}$-norm $|f|_{C^{k}}(x)$ at $x \in \Sigma$ of a smooth function $f \in C^{\infty}(\Sigma, \mathbb{C})$ by using the Levi-Civita connection on $\left(T \Sigma, \omega_{\Sigma}\right)$.

We fix a point $a \in D$ and work in coordinates centered at $a$. Let $\mathfrak{e}_{L}$ be the holomorphic frame of $L$ near $a$ corresponding to the trivialization in the condition $(\alpha)$. By assumptions $(\alpha)$ and $(\beta)$ we have the following identification of the geometric data in the coordinate $z$ on the punctured disc $\mathbb{D}_{4 r}^{*}$ of radius $4 r$ centered at $a$, via the trivialization $\mathfrak{e}_{L}$ of $L$,

$$
\left.\left(\Sigma, \omega_{\Sigma}, L, h\right)\right|_{\mathbb{D}_{4 r}^{*}}=\left.\left(\mathbb{D}^{*}, \omega_{\mathbb{D}^{*}}, \mathbb{C}, h_{\mathbb{D}^{*}}=\left|\log \left(|z|^{2}\right)\right| \cdot h_{0}\right)\right|_{\mathbb{D}_{4 r}^{*}}, \quad \text { with } 0<r<(4 e)^{-1} .
$$

In [5,Theorem 1.2] we proved the following weighted diagonal expansion of the Bergman kernel:

Theorem 1.1 Assume that $\left(\Sigma, \omega_{\Sigma}, L, h\right)$ fulfill conditions $(\alpha)$ and $(\beta)$. Then the following estimate holds: for any $\ell, k \in \mathbb{N}$, and every $\delta>0$, there exists $C=C(\ell, k, \delta)>0$ such that for all $p \in \mathbb{N}^{*}$, and $z \in V_{1} \cup \ldots \cup V_{N}$ with the local coordinate $z_{j}$,

$$
\left|B_{p}-B_{p}^{\mathbb{D}^{*}}\right|_{C^{k}}\left(z_{j}\right) \leq C p^{-\ell}\left|\log \left(\left|z_{j}\right|^{2}\right)\right|^{-\delta},
$$

with norms computed with help of $\omega_{\Sigma}$ and the associated Levi-Civita connection on $\mathbb{D}_{4 r}^{*}$.

Note that in [5,Theorem 1.1] we also established the off-diagonal expansion of the Bergman kernel $B_{p}(\cdot, \cdot)$.

For each $p \geq 2$ fixed $\left(|z|^{2}\left|\log \left(|z|^{2}\right)\right|^{p}\right)^{-1} B_{p}^{\mathbb{D}^{*}}(z)$ is smooth and strictly positive on $\mathbb{D}_{4 r}$, as follows from (2.7). By [5,Remark 3.2], any holomorphic $\boldsymbol{L}^{2}$-section of $L^{p}$ over $\Sigma$ extends to a homomorphic section on $\bar{\Sigma}$ (see the inclusion (1.3)) vanishing at 0 in $\mathbb{D}_{4 r}$. Thus by the formula (1.4) for $B_{p}$ we see that the quotient $\frac{B_{p}}{B_{p}^{\mathbb{D}^{*}}}$ is a smooth function on $\mathbb{D}_{4 r}$ for each $p \geq 2$. 
The main result of the present paper is the following estimate of the quotient of the Bergman kernels from (1.7):

Theorem 1.2 If $\left(\Sigma, \omega_{\Sigma}, L, h\right)$ fulfill conditions $(\alpha)$ and $(\beta)$, then

$$
\sup _{z \in V_{1} \cup \ldots \cup V_{N}}\left|\frac{B_{p}}{B_{p}^{\mathbb{D}^{*}}}(z)-1\right|=\mathcal{O}\left(p^{-\infty}\right),
$$

i.e., for any $\ell>0$ there exists $C>0$ such that for any $p \in \mathbb{N}^{*}$ we have

$$
\sup _{z \in V_{1} \cup \ldots \cup V_{N}}\left|\frac{B_{p}}{B_{p}^{\mathbb{D}^{*}}}(z)-1\right| \leq C p^{-\ell} .
$$

Theorem 1.2 is related to estimates in exponentially small neighborhoods of the punctures obtained in [24,Theorem 1.6] and [25,Lemma 3.3].

Theorem 1.3 For all $k \geq 1$ and $D_{1}, \ldots, D_{k} \in\left\{\frac{\partial}{\partial z}, \frac{\partial}{\partial \bar{z}}\right\}$ we have

$$
\sup _{z \in \overline{V_{1}} \cup \ldots \cup \overline{V_{N}}}\left|\left(D_{1} \cdots D_{k}\right) \frac{B_{p}}{B_{p}^{\mathbb{D}^{*}}}(z)\right|=\mathcal{O}\left(p^{-\infty}\right) .
$$

Remark 1.4 Theorem 1.1 admits a generalization to orbifold Riemann surfaces. Indeed, assume that $\bar{\Sigma}$ is a compact orbifold Riemann surface such that the finite set $D \subset \bar{\Sigma}$ does not meet the (orbifold) singular set of $\bar{\Sigma}$. Then by the same argument as in [5,Remark 1.3] (using $[14,15]$ ) we see that Theorems 1.2 and 1.3 still hold in this context.

Note that the $C^{k}$-norm used in (1.7) is induced by $\omega_{\mathbb{D}^{*}}$, roughly the sup-norm with respect to the derivatives defined by the vector fields $z \log \left(|z|^{2}\right) \frac{\partial}{\partial z}$ and $\bar{z} \log \left(|z|^{2}\right) \frac{\partial}{\partial \bar{z}}$, which vanish at $z=0$. Hence the norm in (1.10) is stronger than the $C^{k}$-norm used in (1.7), because the norm in (1.10) is defined by using derivatives along the vector fields $\frac{\partial}{\partial z}$ and $\frac{\partial}{\partial \bar{z}}$.

Let us mention that the difficulty of the estimates (1.9) and (1.10) consists in the fact that $B_{p}^{\mathbb{D}^{*}}(\cdot)$ takes extremely small values arbitrarily near the origin (this can be seen in $[5, \S 3.2]$ and it is specific to the non-compact framework), thus they don't follow from [5,Theorem 1.2]. What estimate $(1.8)$ says is that $B_{p}(\cdot)$ follows such a behaviour very closely in the corresponding regions of $\Sigma$ via the chosen coordinates.

In order to tackle this difficulty we employ the following strategy to approach Theorems 1.2 and 1.3. We choose a special orthonormal basis $\left\{\sigma_{\ell}^{(p)}\right\}_{\ell=1}^{d_{p}}$ of $H_{(2)}^{0}\left(\Sigma, L^{p}\right)$ starting from $z^{l}$ on $\mathbb{D}_{4 r}^{*}$ for $1 \leq l \leq \delta_{p}$ with $0<\alpha<\delta_{p} / p<\alpha_{1}<1$. Our choice of $\sigma_{\ell}^{(p)}$ implies that the coefficients of the expansion

$$
\sigma_{\ell}^{(p)}(z)=\sum_{j=1}^{\infty} a_{j \ell}^{(p)} z^{j}
$$

of $\sigma_{\ell}^{(p)}$ on $\mathbb{D}_{4 r}^{*}$ satisfy $a_{j \ell}^{(p)}=0$ if $j<\delta_{p}$ and $j<l \leq d_{p}$ (cf. (2.32)). Now we separate the contribution of $\sigma_{\ell}^{(p)}, c_{\ell}^{(p)}$ (cf. (2.6)), $a_{j \ell}^{(p)}$ in $B_{p}, B_{p}^{\mathbb{D}^{*}}$ in two groups: $1 \leq j, \ell \leq \delta_{p}$; $\max \{j, \ell\} \geq \delta_{p}+1$. The contribution corresponding to $1 \leq j, \ell \leq \delta_{p}$, will be controlled by using Lemma 2.1 (or 3.1). The contribution corresponding to $\max \{j, \ell\} \geq \delta_{p}+1$ will be handled by a direct application of Cauchy inequalities (2.23). It turns out that by suitably choosing $c, A>0$ this contribution has uniformly the relative size $2^{-\alpha p}$ compared to $B_{p}^{\mathbb{D}^{*}}$ on $|z| \leq c p^{-A}$. 
This paper is organized as follows. In Sect. 2, we establish Theorem 1.2 based on the off-diagonal expansion of Bergman kernel from [5,§6]. In Sect. 3, we establish Theorem 1.3 by refining the argument from Sect. 2 . In Sect. 4 we give some applications of the main results.

Notation: We denote $\lfloor x\rfloor$ as the integer part of $x \in \mathbb{R}$.

\section{$2 C^{0}$-estimate for the quotient of Bergman kernels}

This section is organized as follows. In Sect. 2.1, we obtain the $C^{0}$-estimate for the quotient of Bergman kernels, Theorem 1.2, admitting first an integral estimate, Lemma 2.1. In Sect. 2.2, we deduce Lemma 2.1 from the two-variable Poincaré type Bergman kernel estimate of [5,Theorem 1.1 and Corollary 6.1].

\subsection{Proof of Theorem 1.2}

We recall first some basic facts. For $\sigma \in C_{0}^{\infty}\left(\Sigma, L^{p}\right)$, the space of smooth and compactly supported sections of $L^{p}$ over $\Sigma$, set

$$
\|\sigma\|_{L_{p}^{2}(\Sigma)}^{2}:=\int_{\Sigma}|\sigma|_{h^{p}}^{2} \omega_{\Sigma}
$$

Let $\boldsymbol{L}_{p}^{2}(\Sigma)$ be the $\|\cdot\|_{\boldsymbol{L}_{p}^{2}(\Sigma)}$-completion of $C_{0}^{\infty}\left(\Sigma, L^{p}\right)$.

By [5,Remark 3.2] the inclusion (1.3) identifies the space $H_{(2)}^{0}\left(\Sigma, L^{p}\right)$ of $\boldsymbol{L}^{2}$-holomorphic sections of $L^{p}$ over $\Sigma$ to the subspace of $H^{0}\left(\bar{\Sigma}, L^{p}\right)$ consisting of sections vanishing at the punctures, so it induces an isomorphism of vector spaces

$$
H_{(2)}^{0}\left(\Sigma, L^{p}\right) \cong H^{0}\left(\bar{\Sigma}, L^{p} \otimes \mathscr{O}_{\bar{\Sigma}}(-D)\right)
$$

where $\mathscr{O}_{\bar{\Sigma}}(-D)$ is the holomorphic line bundle on $\bar{\Sigma}$ defined by the divisor $-D$. By the Riemann-Roch theorem we have for all $p$ with $p \operatorname{deg}(L)-N>2 g-2$,

$$
d_{p}:=\operatorname{dim} H_{(2)}^{0}\left(\Sigma, L^{p}\right)=\operatorname{dim} H^{0}\left(\bar{\Sigma}, L^{p} \otimes \mathscr{O}_{\bar{\Sigma}}(-D)\right)=\operatorname{deg}(L) p+1-g-N,
$$

where $\operatorname{deg}(L)$ is the degree of $L$ over $\bar{\Sigma}$, and $g$ is the genus of $\bar{\Sigma}$.

The Bergman kernel function (1.4) satisfies the following variational characterization, see e.g. [12,Lemma 3.1],

$$
B_{p}(z)=\sup _{0 \neq \sigma \in H_{(2)}^{0}\left(\Sigma, L^{p}\right)} \frac{|\sigma(z)|_{h^{p}}^{2}}{\|\sigma\|_{L_{p}^{2}(\Sigma)}^{2}}, \quad \text { for } z \in \Sigma .
$$

By the expansion of the Bergman kernel on a complete manifold [22,Theorem 6.1.1] (cf. also [5,Theorem 2.1, Corollary 2.4]), there exist coefficients $\boldsymbol{b}_{i} \in C^{\infty}(\Sigma), i \in \mathbb{N}$, such that for any $k, m \in \mathbb{N}$, any compact set $K \subset \Sigma$, we have in the $C^{m}$-topology on $K$,

$$
B_{p}(x)=\sum_{i=0}^{k} \mathbf{b}_{i}(x) p^{1-i}+\mathcal{O}\left(p^{-k}\right), \quad \text { as } p \rightarrow+\infty,
$$

with $\boldsymbol{b}_{0}=-\boldsymbol{b}_{1}=\frac{1}{2 \pi}$ on each $V_{j}$. 
Consider now for $p \geq 2$ the space $H_{(2)}^{p}\left(\mathbb{D}^{*}\right)$ of holomorphic $\boldsymbol{L}^{2}$-functions on $\mathbb{D}^{*}$ with respect to the weight $\|1\|^{2}(z)=\left|\log \left(|z|^{2}\right)\right|^{p}$ (corresponding to a metric on the trivial line bundle $\mathbb{C}$ ) and volume form $\omega_{\mathbb{D}^{*}}$ on $\mathbb{D}^{*}$. An orthonormal basis of $H_{(2)}^{p}\left(\mathbb{D}^{*}\right)$ is given by (cf. [5,Theorem 3.1]),

$$
c_{\ell}^{(p)} z^{\ell} \text { with } \ell \in \mathbb{N}, \ell \geq 1 \text { and } c_{\ell}^{(p)}=\left(\frac{\ell^{p-1}}{2 \pi(p-2) !}\right)^{1 / 2}=\left\|z^{\ell}\right\|_{L_{p}^{2}\left(\mathbb{D}^{*}\right)}^{-1}
$$

and hence

$$
B_{p}^{\mathbb{D}^{*}}(z)=\left|\log \left(|z|^{2}\right)\right|^{p} \sum_{\ell=1}^{\infty}\left(c_{\ell}^{(p)}\right)^{2}|z|^{2 \ell}, \quad \text { for } z \in \mathbb{D}^{*}
$$

For any $m \in \mathbb{N}, 0<b<1$ and $0<\gamma<\frac{1}{2}$ there exists by [5,Proposition 3.3] $\epsilon=\epsilon(b, \gamma)>0$ such that

$$
\left\|B_{p}^{\mathbb{D}^{*}}(z)-\frac{p-1}{2 \pi}\right\|_{C^{m}\left(\left\{b e^{-p^{\gamma}} \leq|z|<1\right\}, \omega_{\mathbb{D}^{*}}\right)}=\mathcal{O}\left(e^{-\epsilon p^{1-2 \gamma}}\right) \text { as } p \rightarrow+\infty .
$$

Taking into account Theorem 1.1 and (2.8) we see that in order to prove Theorem 1.2 it suffices, after reducing to some $V_{j}$ and identifying the geometric data on $\mathbb{D}_{4 r}^{*}$ and $\Sigma$ via (1.6), to show that for some (small) $c>0$ and (large) $A>0$, and for all $l \geq 0$ there exists $C=C(c, A, l)>0$ such that for all $p \geq 2$,

$$
\sup _{0<|z| \leq c p^{-A}}\left|\frac{B_{p}}{B_{p}^{\mathbb{D}^{*}}}(z)-1\right| \leq C p^{-l} .
$$

We now start to establish (2.9). In the whole paper we use the following conventions.

We fix $0<r<(4 e)^{-1}$ as in (1.6), and $0<\beta<1$ such that $r^{\beta}<2 r$.

We fix a (non-increasing) smooth cut-off function $\chi:[0,1] \rightarrow \mathbb{R}$,

satisfying $\chi(u)=1$ if $u \leq r^{\beta}$ and $\chi(u)=0$ if $u \geq 2 r$.

We set $\delta_{p}=\left\lfloor\frac{p-2}{2|\log r|}\right\rfloor$ for $p \in \mathbb{N}, p \geq 2$.

The choice of $\delta_{p}$ will become clear in (2.19), (2.27) and (2.41), for example. By (2.10) there exist $\alpha>0$ such that

$$
\alpha p \leq \delta_{p} \quad \text { and } \quad \delta_{p}+1 \leq \frac{1}{2} p \quad \text { for } p \geq 2+2|\log r| .
$$

To establish (2.9) we proceed along the following lines:

1. For $\ell \in\left\{1, \ldots, \delta_{p}\right\}$, we set

$$
\phi_{\ell, 0}^{(p)}=c_{\ell}^{(p)} \chi(|z|) z^{\ell}
$$

2. Using the trivialization, that is, identifying $\phi_{\ell, 0}^{(p)}$ with $\phi_{\ell, 0}^{(p)} \mathfrak{e}_{L}^{p}$ when we work on $\Sigma$, we see the $\phi_{\ell, 0}^{(p)}$ as (smooth) $\boldsymbol{L}^{2}$ sections of $L^{p}$ over $\Sigma$, that we correct into holomorphic $\boldsymbol{L}^{2}$ sections $\phi_{\ell}^{(p)}$ of $L^{p}$, by orthogonal $\boldsymbol{L}_{p}^{2}(\Sigma)$-projection. 
3. Next we correct the family $\left(\phi_{\ell}^{(p)}\right)_{1 \leq \ell \leq \delta_{p}}$ into an orthonormal family $\left(\sigma_{\ell}^{(p)}\right)_{1 \leq \ell \leq \delta_{p}}$ by the Gram-Schmidt procedure, and we further complete $\left(\sigma_{\ell}^{(p)}\right)_{1 \leq \ell \leq \delta_{p}}$ into an orthonormal basis $\left(\sigma_{\ell}^{(p)}\right)_{1 \leq \ell \leq d_{p}}$ of $H_{(2)}^{0}\left(\Sigma, L^{p}\right)$. In particular, for any $1 \leq j \leq \delta_{p}$,

$$
\operatorname{Span}\left\{\phi_{1,0}^{(p)}, \cdots, \phi_{j, 0}^{(p)}\right\}=\operatorname{Span}\left\{\phi_{1}^{(p)}, \cdots, \phi_{j}^{(p)}\right\}=\operatorname{Span}\left\{\sigma_{1}^{(p)}, \cdots, \sigma_{j}^{(p)}\right\} .
$$

4. Finally, we carefully compare $B_{p}^{\mathbb{D}^{*}}$ with $B_{p}$ using the three steps of the above construction to get estimate (2.9); of particular importance are the following intermediate estimates which will be deduced from $[5, \S 6]$ :

Lemma 2.1 With the notations above, for all $m \in \mathbb{N}$, there exists $C=C(m)>0$ such that for all $p \in \mathbb{N}^{*}, p \geq 2$, and all $j, \ell \in\left\{1, \ldots, \delta_{p}\right\}$,

$$
\begin{aligned}
1-C p^{-m} \leq\left\|\phi_{\ell, 0}^{(p)}\right\|_{L_{p}^{2}(\Sigma)}^{2} & =\left(c_{\ell}^{(p)}\right)^{2} \int_{\mathbb{D}_{2 r}^{*}} \chi^{2}(|z|)|z|^{2 \ell}\left|\log \left(|z|^{2}\right)\right|^{p} \omega_{\mathbb{D}^{*}} \\
& \leq\left(c_{\ell}^{(p)}\right)^{2} \int_{\mathbb{D}_{2 r}^{*}} \chi(|z|)|z|^{2 \ell}\left|\log \left(|z|^{2}\right)\right|^{p} \omega_{\mathbb{D}^{*}} \leq 1,
\end{aligned}
$$

and moreover,

$$
\begin{aligned}
& \left\|\sigma_{\ell}^{(p)}-\phi_{\ell, 0}^{(p)}\right\|_{L_{p}^{2}(\Sigma)} \leq C p^{-m}, \\
& \quad\left|\left\langle\phi_{j}^{(p)}, \sigma_{\ell}^{(p)}\right\rangle_{L_{p}^{2}(\Sigma)}-\delta_{j \ell}\right| \leq C p^{-m} .
\end{aligned}
$$

The proof of Lemma 2.1 is postponed to Sect. 2.2.

Notice that we take care of stating estimates uniform in $j, \ell \in\left\{1, \ldots, \delta_{p}\right\}$. Observe moreover that (2.14), (2.15) are integral estimates, whereas we want to establish pointwise estimates in the end, hence we need an extra effort to convert these (among others) into (2.9).

Let us see now how to build on (2.15) to get the desired (2.9).

First, by (1.4), (2.7), (2.12), and the construction of $\phi_{\ell, 0}^{(p)}$ and $\sigma_{\ell}^{(p)}$ we have for $z \in \mathbb{D}_{r}^{*}$,

$$
\begin{aligned}
B_{p}^{\mathbb{D}^{*}}(z)= & \sum_{\ell=1}^{\delta_{p}}\left|\phi_{\ell, 0}^{(p)}\right|_{h^{p}, z}^{2}+\left|\log \left(|z|^{2}\right)\right|^{p} \sum_{\ell=\delta_{p}+1}^{\infty}\left(c_{\ell}^{(p)}\right)^{2}|z|^{2 \ell} \\
= & B_{p}(z)-\sum_{\ell=\delta_{p}+1}^{d_{p}}\left|\sigma_{\ell}^{(p)}\right|_{h^{p}, z}^{2}+2 \operatorname{Re}\left[\sum_{\ell=1}^{\delta_{p}}\left\langle\sigma_{\ell}^{(p)}, \phi_{\ell, 0}^{(p)}-\sigma_{\ell}^{(p)}\right\rangle_{h^{p}, z}\right] \\
& +\sum_{\ell=1}^{\delta_{p}}\left|\phi_{\ell, 0}^{(p)}-\sigma_{\ell}^{(p)}\right|_{h^{p}, z}^{2}+\left|\log \left(|z|^{2}\right)\right|^{p} \sum_{\ell=\delta_{p}+1}^{\infty}\left(c_{\ell}^{(p)}\right)^{2}|z|^{2 \ell} .
\end{aligned}
$$

We deal with the summands of the last three terms separately; we start by claiming that up to a judicious choice of $c>0$ and $A>0$ we have for $0<|z| \leq c p^{-A}$ :

$$
\left|\log \left(|z|^{2}\right)\right|^{p} \sum_{\ell=\delta_{p}+1}^{\infty}\left(c_{\ell}^{(p)}\right)^{2}|z|^{2 \ell}=\mathcal{O}\left(p^{-\infty}\right) \cdot B_{p}^{\mathbb{D}^{*}}(z), \quad \text { as } p \rightarrow \infty
$$

that is,

$$
\sup _{0<|z| \leq c p^{-A}}\left[B_{p}^{\mathbb{D}^{*}}(z)^{-1}\left|\log \left(|z|^{2}\right)\right|^{p} \sum_{\ell=\delta_{p}+1}^{\infty}\left(c_{\ell}^{(p)}\right)^{2}|z|^{2 \ell}\right]=\mathcal{O}\left(p^{-\infty}\right) .
$$


Indeed, we have $\frac{\ell+\delta_{p}}{\ell} \leq \delta_{p}+1$ for all $\ell \geq 1$, so by (2.6) we have for $z \in \mathbb{D}^{*}$,

$$
\begin{aligned}
\left|\log \left(|z|^{2}\right)\right|^{p} \sum_{\ell=\delta_{p}+1}^{\infty}\left(c_{\ell}^{(p)}\right)^{2}|z|^{2 \ell} & =\left|\log \left(|z|^{2}\right)\right|^{p} \frac{|z|^{2 \delta_{p}}}{2 \pi(p-2) !} \sum_{\ell=1}^{\infty}\left(\frac{\ell+\delta_{p}}{\ell}\right)^{p-1} \ell^{p-1}|z|^{2 \ell} \\
& \leq\left(\delta_{p}+1\right)^{p-1} \frac{|z|^{2 \delta_{p}}}{2 \pi(p-2) !}\left|\log \left(|z|^{2}\right)\right|^{p} \sum_{\ell=1}^{\infty} \ell^{p-1}|z|^{2 \ell} \\
& =\left(\delta_{p}+1\right)^{p-1}|z|^{2 \delta_{p}} B_{p}^{\mathbb{D}^{*}}(z)
\end{aligned}
$$

From (2.11) follows

$$
\left(\delta_{p}+1\right)|z|^{2 \delta_{p} /(p-1)} \leq \frac{1}{2} p|z|^{2 \alpha} \leq \frac{1}{2}, \quad \text { for all }|z| \leq p^{-1 /(2 \alpha)} .
$$

From (2.18) and (2.19) we get (2.17) with $c=r$ and $A=\frac{1}{2 \alpha}$.

In the similar vein we now show the following.

Lemma 2.2 For $c=r$ and $A=\frac{1}{2 \alpha}$, with $\alpha$ satisfying (2.11), we have uniformly in $z \in \mathbb{D}_{c p^{-A}}^{*}$,

$$
\sum_{\ell=\delta_{p}+1}^{d_{p}}\left|\sigma_{\ell}^{(p)}\right|_{h^{p}, z}^{2}=\mathcal{O}\left(p^{-\infty}\right) \cdot B_{p}^{\mathbb{D}^{*}}(z) .
$$

We have uniformly in $z \in \mathbb{D}_{c p^{-A}}^{*}$, and $\ell \in\left\{1, \ldots, \delta_{p}\right\}$,

$$
\left|\sigma_{\ell}^{(p)}-\phi_{\ell, 0}^{(p)}\right|_{h^{p}, z}=\mathcal{O}\left(p^{-\infty}\right) \cdot B_{p}^{\mathbb{D}^{*}}(z)^{1 / 2} .
$$

Proof Let $p \geq 2, \ell \in\left\{1, \cdots, d_{p}\right\}$. By [5,Remark 3.2] and (1.3) we know that $\sigma_{\ell}^{(p)}$ is a holomorphic section of $L^{p}$ over $\bar{\Sigma}$ vanishing at $D$. We use the trivialization (1.6) to set

$$
\sigma_{\ell}^{(p)}=\left(\sum_{j=1}^{\infty} a_{j \ell}^{(p)} z^{j}\right) \mathfrak{e}_{L}^{p}=: s_{\ell}^{(p)} \mathfrak{e}_{L}^{p} \quad \text { on } \mathbb{D}_{4 r}^{*} .
$$

We have for $j \geq 1$ by (1.4), (1.6), (2.5), (2.22) and Cauchy inequalities,

$$
\begin{aligned}
\left|a_{j \ell}^{(p)}\right| & \leq(2 r)^{-j} \sup _{|z|=2 r}\left|s_{\ell}^{(p)}(z)\right| \\
& =(2 r)^{-j}\left|\log \left(|2 r|^{2}\right)\right|^{-p / 2} \sup _{|z|=2 r}\left|\sigma_{\ell}^{(p)}(z)\right|_{h^{p}} \\
& \leq(2 r)^{-j}\left|\log \left(|2 r|^{2}\right)\right|^{-p / 2} \sup _{|z|=2 r} B_{p}(z)^{1 / 2} \\
& \leq C p^{1 / 2}(2 r)^{-j}\left|\log \left(|2 r|^{2}\right)\right|^{-p / 2} .
\end{aligned}
$$

Thus by (1.6) and (2.23) we have for $z \in \mathbb{D}_{r}^{*}$,

$$
\begin{aligned}
\left|\sum_{j=\delta_{p}+1}^{\infty} a_{j \ell}^{(p)} z^{j}\right|_{h^{p}} & \leq C\left|\log \left(|z|^{2}\right)\right|^{p / 2} \sum_{j=\delta_{p}+1}^{\infty} p^{1 / 2}\left|\log \left(|2 r|^{2}\right)\right|^{-p / 2}\left(\frac{|z|}{2 r}\right)^{j} \\
& =C p^{1 / 2}\left(\frac{\left|\log \left(|z|^{2}\right)\right|}{\left|\log \left(|2 r|^{2}\right)\right|}\right)^{p / 2}\left(1-\frac{|z|}{2 r}\right)^{-1}\left(\frac{|z|}{2 r}\right)^{\delta_{p}+1} .
\end{aligned}
$$


By (2.6) and (2.7) we have

$$
\left|\log \left(|z|^{2}\right)\right|^{p / 2}|z|=|z|_{h_{\mathbb{D}^{*}}^{p}} \leq\|z\|_{L_{p}^{2}\left(\mathbb{D}^{*}\right)} B_{p}^{\mathbb{D}^{*}}(z)^{1 / 2}=(2 \pi(p-2) !)^{1 / 2} B_{p}^{\mathbb{D}^{*}}(z)^{1 / 2} .
$$

We deduce from (2.24) and (2.25) that there exists $C>0$ such that the following estimate holds uniformly in $\ell \in\left\{1, \ldots, d_{p}\right\},|z| \in \mathbb{D}_{r}^{*}$,

$$
\left|\sum_{j=\delta_{p}+1}^{\infty} a_{j \ell}^{(p)} z^{j}\right|_{h^{p}} \leq C p^{-1 / 2}\left(\left(\frac{|z|}{2 r}\right)^{2 \delta_{p} / p} \frac{(p !)^{1 / p}}{\left|\log \left(|2 r|^{2}\right)\right|}\right)^{p / 2} B_{p}^{\mathbb{D}^{*}}(z)^{1 / 2} .
$$

By (2.11) we have for $A=\frac{1}{2 \alpha}, c_{0}=r e^{1 /(2 \alpha)}\left|\log \left(|2 r|^{2}\right)\right|^{1 /(2 \alpha)}>r$, and $p \gg 1$,

$$
\left(\frac{|z|}{2 r}\right)^{2 \delta_{p} / p} \frac{1}{\left|\log \left(|2 r|^{2}\right)\right|} \leq\left(\frac{|z|}{2 r}\right)^{2 \alpha} \frac{1}{\left|\log \left(|2 r|^{2}\right)\right|} \leq 2^{-2 \alpha} \frac{e}{p}, \quad \text { for }|z| \leq c_{0} p^{-A} .
$$

Recall that the Stirling formula states

$$
\frac{p^{p}}{p !}=(2 \pi p)^{-1 / 2} e^{p}\left(1+\mathcal{O}\left(p^{-1}\right)\right) \quad \text { as } p \rightarrow+\infty .
$$

We infer from (2.26), (2.27) and (2.28), that there exists $C>0$ such that the following estimate holds uniformly in $|z| \leq r p^{-A}$ and $\ell \in\left\{1, \ldots, d_{p}\right\}$,

$$
\left|\sum_{j=\delta_{p}+1}^{\infty} a_{j \ell}^{(p)} z^{j}\right|_{h^{p}} \leq C 2^{-p \alpha} B_{p}^{\mathbb{D}^{*}}(z)^{1 / 2}
$$

Note that $\phi_{j}^{(p)}-\phi_{j, 0}^{(p)}$ is orthogonal to $H_{(2)}^{0}\left(\Sigma, L^{p}\right)$. By (2.1), (2.12), (2.22), and since $\sigma_{\ell}^{(p)}$ are holomorphic, we have for $j \in\left\{1, \cdots, \delta_{p}\right\}, \ell \in\left\{1, \cdots, d_{p}\right\}$,

$$
\left\langle\sigma_{\ell}^{(p)}, \phi_{j}^{(p)}\right\rangle_{\boldsymbol{L}_{p}^{2}(\Sigma)}=\left\langle\sigma_{\ell}^{(p)}, \phi_{j, 0}^{(p)}\right\rangle_{\boldsymbol{L}_{p}^{2}(\Sigma)}=c_{j}^{(p)} a_{j \ell}^{(p)} \int_{\mathbb{D}_{2 r}^{*}} \chi(|z|)\left|z^{j}\right|^{2}\left|\log \left(|z|^{2}\right)\right|^{p} \omega_{\mathbb{D}^{*}}
$$

By (2.13) we have

$$
\left\langle\sigma_{\ell}^{(p)}, \phi_{j}^{(p)}\right\rangle_{L_{p}^{2}(\Sigma)}=0 \text { for } j \in\left\{1, \cdots, \delta_{p}\right\}, j<\ell .
$$

From (2.30) and (2.31) we get

$$
a_{j \ell}^{(p)}=0 \quad \text { for } j \in\left\{1, \cdots, \delta_{p}\right\}, \ell \in\left\{\delta_{p}+1, \cdots, d_{p}\right\} .
$$

By (2.3), (2.29) and (2.32), we get (2.20).

Fixing $\ell \in\left\{1, \ldots, \delta_{p}\right\}$, we have on $\mathbb{D}_{r}^{*}$ by (2.10), (2.12), (2.22),

$$
\left(\sigma_{\ell}^{(p)}-\phi_{\ell, 0}^{(p)} \mathfrak{e}_{L}^{p}\right)(z)=\left(\left(a_{\ell \ell}^{(p)}-c_{\ell}^{(p)}\right) z^{\ell}+\sum_{\substack{j=1 \\ j \neq \ell}}^{\infty} a_{j \ell}^{(p)} z^{j}\right) \mathfrak{e}_{L}^{p} .
$$

From Lemma 2.1 and (2.30) we have uniformly for $j, \ell \in\left\{1, \ldots, \delta_{p}\right\}$,

$$
\begin{aligned}
a_{j \ell}^{(p)} & =c_{j}^{(p)}\left(\left(c_{j}^{(p)}\right)^{2} \int_{\mathbb{D}_{2 r}^{*}} \chi(|z|)\left|z^{j}\right|^{2}\left|\log \left(|z|^{2}\right)\right|^{p} \omega_{\mathbb{D}^{*}}\right)^{-1}\left\langle\sigma_{\ell}^{(p)}, \phi_{j}^{(p)}\right\rangle_{\boldsymbol{L}_{p}^{2}(\Sigma)} \\
& =\left(\delta_{j \ell}+\mathcal{O}\left(p^{-\infty}\right)\right) c_{j}^{(p)} .
\end{aligned}
$$


Thus from (2.7), (2.34) we have on $\mathbb{D}_{r}^{*}$ uniformly in $\ell \in\left\{1, \ldots, \delta_{p}\right\}$,

$$
\begin{aligned}
& \left|\left(\left(a_{\ell \ell}^{(p)}-c_{\ell}^{(p)}\right) z^{\ell}+\sum_{\substack{j=1 \\
j \neq \ell}}^{\delta_{p}} a_{j \ell}^{(p)} z^{j}\right) \mathfrak{e}_{L}^{p}\right|_{h^{p}}^{2}=\left|\log \left(|z|^{2}\right)\right|^{p}\left|\sum_{j=1}^{\delta_{p}}\left(a_{j \ell}^{(p)}-\delta_{j \ell} c_{j}^{(p)}\right) z^{j}\right|^{2} \\
& \quad \leq \mathcal{O}\left(p^{-\infty}\right)\left|\log \left(|z|^{2}\right)\right|^{p} \delta_{p} \sum_{j=1}^{\delta_{p}}\left(c_{j}^{(p)}\right)^{2}|z|^{2 j} \leq \delta_{p} \mathcal{O}\left(p^{-\infty}\right) B_{p}^{\mathbb{D}^{*}}(z)
\end{aligned}
$$

Now $\delta_{p}$ can be absorbed in the factor $\mathcal{O}\left(p^{-\infty}\right)$, since $\delta_{p}=\mathcal{O}(p)$ by (2.10). Combining (2.29) with (2.35) we conclude that (2.21) holds uniformly in $\ell \in\left\{1, \ldots, \delta_{p}\right\}$.

Since $\delta_{p}=\mathcal{O}(p)$ and $\left|\sigma_{\ell}^{(p)}\right|_{h^{p}, z} \leq B_{p}(z)^{1 / 2},(2.21)$ also yields

$$
\left|\sum_{\ell=1}^{\delta_{p}}\left\langle\sigma_{\ell}^{(p)}, \phi_{\ell, 0}^{(p)}-\sigma_{\ell}^{(p)}\right\rangle_{h^{p}, z}\right|=\mathcal{O}\left(p^{-\infty}\right) \cdot B_{p}^{\mathbb{D}^{*}}(z)^{1 / 2} B_{p}(z)^{1 / 2} \quad \text { on } \mathbb{D}_{c p^{-A}}^{*} .
$$

This way, putting together (2.16), (2.17), (2.20), (2.21) and (2.36), we obtain

$$
\left(1+\mathcal{O}\left(p^{-\infty}\right)\right) \cdot B_{p}^{\mathbb{D}^{*}}(z)=B_{p}(z)+\mathcal{O}\left(p^{-\infty}\right) \cdot B_{p}^{\mathbb{D}^{*}}(z)^{1 / 2} B_{p}(z)^{1 / 2} \quad \text { on } \mathbb{D}_{c p^{-A}}^{*},
$$

and this implies (2.9). The proof of Theorem 1.2 is completed.

\subsection{Proof of Lemma 2.1}

At first, as $0 \leq \chi \leq 1$ and $\operatorname{supp}(\chi) \subset \mathbb{D}_{2 r}^{*}$, we get from (1.6), (2.6) and (2.10),

$$
\begin{aligned}
& \left\|\phi_{\ell, 0}^{(p)}\right\|_{L_{p}^{2}(\Sigma)}^{2}=\left\|\phi_{\ell, 0}^{(p)}\right\|_{L_{p}^{2}\left(\mathbb{D}^{*}\right)}^{2} \leq\left(c_{\ell}^{(p)}\right)^{2} \int_{\mathbb{D}^{*}} \chi(|z|)\left|\log \left(|z|^{2}\right)\right|^{p}|z|^{2 \ell} \omega_{\mathbb{D}^{*}} \\
& \leq\left(c_{\ell}^{(p)}\right)^{2} \int_{\mathbb{D}^{*}}\left|\log \left(|z|^{2}\right)\right|^{p}|z|^{2 \ell} \omega_{\mathbb{D}^{*}}=\left\|c_{\ell}^{(p)} z^{\ell}\right\|_{L_{p}^{2}\left(\mathbb{D}^{*}\right)}^{2}=1 .
\end{aligned}
$$

This implies the inequalities of the right-hand side of (2.14).

We establish now the lower bound of (2.14). For $\ell \in\left\{1, \ldots, \delta_{p}\right\}$ we have by (1.1), (2.6), (2.10) and (2.12),

$$
\begin{aligned}
1-\left\|\phi_{\ell, 0}^{(p)}\right\|_{\boldsymbol{L}_{p}^{2}\left(\mathbb{D}^{*}\right)}^{2} & =\left(c_{\ell}^{(p)}\right)^{2} \int_{\mathbb{D}^{*}}\left|\log \left(|z|^{2}\right)\right|^{p}\left\{1-\chi^{2}(|z|)\right\}|z|^{2 \ell} \omega_{\mathbb{D}^{*}} \\
& =\frac{\ell^{p-1}}{(p-2) !} \int_{r^{\beta}}^{1}\left|\log \left(t^{2}\right)\right|^{p} t^{2 \ell}\left(1-\chi^{2}(t)\right) \frac{2 t d t}{t^{2}\left|\log \left(t^{2}\right)\right|^{2}} \\
& \stackrel{u=-2 \ell \log t}{=} \frac{1}{(p-2) !} \int_{0}^{2 \ell \beta|\log r|} u^{p-2} e^{-u}\left(1-\chi^{2}\left(e^{-u /(2 \ell)}\right)\right) d u \\
& \leq \frac{1}{(p-2) !} \int_{0}^{2 \delta_{p} \beta|\log r|} u^{p-2} e^{-u} d u .
\end{aligned}
$$

The function $u \mapsto \log u-u$ is strictly increasing on $(0,1]$ and equals -1 at $u=1$, hence

$$
\log \beta-\beta<-1 .
$$


As $u^{p-2} e^{-u}$ is strictly increasing on [0,p-2], and $2 \delta_{p}|\log r| \leq p-2$ (by (2.10)), so (2.28) and (2.40) imply

$$
\begin{aligned}
& \frac{1}{(p-2) !} \int_{0}^{2 \delta_{p} \beta|\log r|} u^{p-2} e^{-u} d u \leq \frac{1}{(p-2) !} \int_{0}^{(p-2) \beta} u^{p-2} e^{-u} d u \\
& \leq \frac{(p-2)^{p-2}}{(p-2) !} e^{(p-2)(\log \beta-\beta)}(p-2) \beta \\
& =\left(\frac{p-2}{2 \pi}\right)^{1 / 2} \beta\left(1+\mathcal{O}\left(p^{-1}\right)\right) e^{(p-2)(\log \beta-\beta+1)} \\
& =\mathcal{O}\left(p^{-\infty}\right) .
\end{aligned}
$$

Combining (2.39) and (2.41) we obtain that the first inequality of (2.14) holds uniformly in $\ell \in\left\{1, \ldots, \delta_{p}\right\}$.

We move on to (2.15) and we first estimate $\left\|\phi_{\ell}^{(p)}-\phi_{\ell, 0}^{(p)}\right\|_{L^{2}\left(\mathbb{D}_{3 r}^{*}\right)}$. Using the identification (1.6) as in $[5,(6.1)]$ we denote for $x, y \in \mathbb{D}_{4 r}^{*}$,

$$
\begin{aligned}
& B_{p}(x, y)=\left|\log \left(|y|^{2}\right)\right|^{p} \beta_{p}^{\Sigma}(x, y), \\
& B_{p}^{\mathbb{D}^{*}}(x, y)=\left|\log \left(|y|^{2}\right)\right|^{p} \beta_{p}^{\mathbb{D}^{*}}(x, y) \text { with } \beta_{p}^{\mathbb{D}^{*}}(x, y)=\frac{1}{2 \pi(p-2) !} \sum_{\ell=1}^{\infty} \ell^{p-1} x^{\ell} \bar{y}^{\ell} .
\end{aligned}
$$

For $\ell \in\left\{1, \ldots, \delta_{p}\right\}$ set

$$
\begin{aligned}
& I_{1, \ell}^{(p)}(x)=\int_{y \in \mathbb{D}_{2 r}^{*}}\left|\log \left(|y|^{2}\right)\right|^{p}\left\{\beta_{p}^{\Sigma}(x, y)-\beta_{p}^{\mathbb{D}^{*}}(x, y)\right\} \chi(|y|) y^{\ell} \omega_{\mathbb{D}^{*}}(y), \\
& I_{2, \ell}^{(p)}(x)=\int_{y \in \mathbb{D}^{*}}\left|\log \left(|y|^{2}\right)\right|^{p} \beta_{p}^{\mathbb{D}^{*}}(x, y)\{\chi(|y|)-1\} y^{\ell} \omega_{\mathbb{D}^{*}}(y), \\
& I_{3, \ell}^{(p)}(x)=\int_{y \in \mathbb{D}^{*}}\left|\log \left(|y|^{2}\right)\right|^{p} \beta_{p}^{\mathbb{D}^{*}}(x, y) y^{\ell} \omega_{\mathbb{D}^{*}}(y)=x^{\ell}
\end{aligned}
$$

where the last equality is a consequence of the reproducing property of the Bergman kernel $B_{p}^{\mathbb{D}^{*}}(\cdot, \cdot)$. By the construction of $\phi_{\ell}^{(p)},(2.10)$, and the reproducing property of $B_{p}(\cdot, \cdot)$ we have for $x \in \mathbb{D}_{4 r}^{*}$,

$$
\begin{aligned}
\phi_{\ell}^{(p)}(x) & =\left(B_{p} \phi_{\ell, 0}^{(p)}\right)(x)=\int_{y \in \Sigma} B_{p}(x, y) \phi_{\ell, 0}^{(p)}(y) \omega_{\Sigma}(y) \\
& =c_{\ell}^{(p)} \int_{y \in \mathbb{D}_{2 r}^{*}}\left|\log \left(|y|^{2}\right)\right|^{p} \beta_{p}^{\Sigma}(x, y) \chi(|y|) y^{\ell} \omega_{\mathbb{D}^{*}}(y) \\
& =c_{\ell}^{(p)}\left(I_{1, \ell}^{(p)}(x)+I_{2, \ell}^{(p)}(x)+I_{3, \ell}^{(p)}(x)\right) .
\end{aligned}
$$


Now [5,Theorem 1.1 or (6.23)] and (2.6) yield for fixed $v>0$ and $m>0$ and for any $x \in \mathbb{D}_{4 r}^{*}, p \geq 2$,

$$
\begin{aligned}
\left|I_{1, \ell}^{(p)}(x)\right| \leq & C(m, v) p^{-m}\left|\log \left(|x|^{2}\right)\right|^{-v-p / 2} \int_{y \in \mathbb{D}_{2 r}^{*}}\left|\log \left(|y|^{2}\right)\right|^{-v+p / 2} \chi(|y|)|y|^{\ell} \omega_{\mathbb{D}^{*}}(y) \\
\leq & C(m, v) p^{-m}\left|\log \left(|x|^{2}\right)\right|^{-v-p / 2} \\
& \cdot\left(\int_{\mathbb{D}^{*}}\left|\log \left(|y|^{2}\right)\right|^{p}|y|^{2 \ell} \omega_{\mathbb{D}^{*}}(y)\right)^{1 / 2}\left(\int_{\mathbb{D}^{*}}\left|\log \left(|y|^{2}\right)\right|^{-2 v} \chi^{2}(|y|) \omega_{\mathbb{D}^{*}}(y)\right)^{1 / 2} \\
= & C^{\prime}(m, v) p^{-m}\left|\log \left(|x|^{2}\right)\right|^{-v-p / 2}\left(c_{\ell}^{(p)}\right)^{-1} .
\end{aligned}
$$

Keeping $v$ fixed and varying $m$ in (2.45) we obtain the following uniform estimate in $\ell \in$ $\left\{1, \ldots, \delta_{p}\right\}$,

$$
\left\|c_{\ell}^{(p)} I_{1, \ell}^{(p)}\right\|_{L_{p}^{2}\left(\mathbb{D}_{3 r}^{*}\right)}=\mathcal{O}\left(p^{-\infty}\right) .
$$

By circle symmetry first and (2.6), (2.14), (2.42) and (2.43) we obtain,

$$
I_{2, \ell}^{(p)}(x)=\left(c_{\ell}^{(p)}\right)^{2}\left[\int_{y \in \mathbb{D}^{*}}\left|\log \left(|y|^{2}\right)\right|^{p}\{\chi(|y|)-1\}|y|^{2 \ell} \omega_{\mathbb{D}^{*}}(y)\right] x^{\ell}=\mathcal{O}\left(p^{-\infty}\right) \cdot x^{\ell},
$$

uniformly in $\ell \in\left\{1, \ldots, \delta_{p}\right\}$. Since $\left\|c_{\ell}^{(p)} x^{\ell}\right\|_{L_{p}^{2}\left(\mathbb{D}_{3 r}^{*}\right)} \leq\left\|c_{\ell}^{(p)} x^{\ell}\right\|_{L_{p}^{2}\left(\mathbb{D}^{*}\right)}=1$, this tells us already that

$$
\left\|c_{\ell}^{(p)} I_{2, \ell}^{(p)}\right\|_{L_{p}^{2}\left(\mathbb{D}_{3 r}^{*}\right)}=\mathcal{O}\left(p^{-\infty}\right) .
$$

Since $0 \leq 1-\chi \leq 1$ and $1-\chi(t)=0$ for $t \leq r^{\beta}$, we get by (2.6), (2.41) and (2.43), as in (2.39), that for $\ell \in\left\{1, \ldots, \delta_{p}\right\}$ the following holds,

$$
\begin{aligned}
& \left\|c_{\ell}^{(p)} I_{3, \ell}^{(p)}(x)-\phi_{\ell, 0}^{(p)}(x)\right\|_{L_{p}^{2}\left(\mathbb{B}_{3 r}^{*}\right)}^{2} \leq\left\|c_{\ell}^{(p)}(1-\chi(|x|)) x^{\ell}\right\|_{L_{p}^{2}\left(\mathbb{D}^{*}\right)}^{2} \\
& =\frac{\ell^{p-1}}{(p-2) !} \int_{r^{\beta}}^{1}\left|\log \left(t^{2}\right)\right|^{p} t^{2 \ell}(1-\chi(t))^{2} \frac{2 t d t}{t^{2}\left|\log \left(t^{2}\right)\right|^{2}} \\
& u=-\frac{2 \ell \log t}{=} \frac{1}{(p-2) !} \int_{0}^{2 \ell \beta|\log r|} u^{p-2} e^{-u}\left(1-\chi\left(e^{-u /(2 \ell)}\right)\right)^{2} d u \\
& \leq \frac{1}{(p-2) !} \int_{0}^{2 \delta_{p} \beta|\log r|} u^{p-2} e^{-u} d u=\mathcal{O}\left(p^{-\infty}\right) .
\end{aligned}
$$

By (2.44), (2.46), (2.48) and (2.49) we get the following estimate uniformly in $\ell \in$ $\left\{1, \ldots, \delta_{p}\right\}$,

$$
\left\|\phi_{\ell, 0}^{(p)}-\phi_{\ell}^{(p)}\right\|_{L_{p}^{2}\left(\mathbb{D}_{3 r}^{*}\right)}=\mathcal{O}\left(p^{-\infty}\right) .
$$

A weak form of [5,Corollary 6.1] tells us that for any $k \in \mathbb{N}, \varepsilon>0$, there exists $C>0$ such that

$$
\left|B_{p}(x, y)\right| \leq C p^{-k} \text { for } d(x, y)>\varepsilon, \quad p \geq 2 .
$$


By (2.38), (2.44) and (2.51),

$$
\left\|\phi_{\ell}^{(p)}\right\|_{L_{p}^{2}\left(\Sigma \backslash \mathbb{D}_{3 r}^{*}\right)}^{2} \leq C p^{-2 k} \int_{\Sigma \backslash \mathbb{D}_{3 r}^{*}} \omega_{\Sigma} \int_{\mathbb{D}_{2 r}^{*}}\left|\phi_{\ell, 0}^{p}(y)\right|_{h^{p}}^{2} \omega_{\Sigma}(y) \leq C p^{-2 k} \int_{\Sigma} \omega_{\Sigma}
$$

From (2.50) and (2.52) we have uniformly in $\ell \in\left\{1, \ldots, \delta_{p}\right\}$,

$$
\left\|\phi_{\ell}^{(p)}-\phi_{\ell, 0}^{(p)}\right\|_{L_{p}^{2}(\Sigma)}^{2}=\left\|\phi_{\ell}^{(p)}-\phi_{\ell, 0}^{(p)}\right\|_{L_{p}^{2}\left(\mathbb{D}_{3 r}^{*}\right)}^{2}+\left\|\phi_{\ell}^{(p)}\right\|_{\boldsymbol{L}_{p}^{2}\left(\Sigma \backslash \mathbb{D}_{3 r}^{*}\right)}^{2}=\mathcal{O}\left(p^{-\infty}\right) .
$$

By (2.14) and (2.53), as $\phi_{j}^{(p)}-\phi_{j, 0}^{(p)}$ is orthogonal to $H_{(2)}^{0}\left(\Sigma, L^{p}\right)$, we have uniformly in $j, \ell \in\left\{1, \ldots, \delta_{p}\right\}$,

$$
\begin{aligned}
\left\langle\phi_{j}^{(p)}, \phi_{\ell}^{(p)}\right\rangle_{L_{p}^{2}(\Sigma)} & =\left\langle\phi_{j, 0}^{(p)}, \phi_{\ell}^{(p)}\right\rangle_{L_{p}^{2}(\Sigma)} \\
& =\left\langle\phi_{j, 0}^{(p)}, \phi_{\ell, 0}^{(p)}\right\rangle_{L_{p}^{2}\left(\mathbb{D}_{2 r}^{*}\right)}+\left\langle\phi_{j, 0}^{(p)}, \phi_{\ell}^{(p)}-\phi_{\ell, 0}^{(p)}\right\rangle_{L_{p}^{2}(\Sigma)} \\
& =\delta_{j \ell}+\mathcal{O}\left(p^{-\infty}\right) .
\end{aligned}
$$

Note that the circle symmetry and (2.12) imply that $\left\langle\phi_{j, 0}^{(p)}, \phi_{\ell, 0}^{(p)}\right\rangle_{L_{p}^{2}\left(\mathbb{D}_{2 r}^{*}\right)}=0$ if $j \neq \ell$. We now observe that the Gram-Schmidt orthonormalization $\left(\sigma_{\ell}^{(p)}\right)_{1 \leq \ell \leq \delta_{p}}$ of the "almostorthonormal" family $\left(\phi_{\ell}^{(p)}\right)_{1 \leq \ell \leq \delta_{p}}$ is the normalization of

$$
\sigma_{\ell}^{\prime(p)}=\phi_{\ell}^{(p)}-\sum_{k=1}^{\ell-1} \frac{\left\langle\phi_{\ell}^{(p)}, \phi_{k}^{(p)}\right\rangle_{L_{p}^{2}(\Sigma)}}{\left\langle\phi_{k}^{(p)}, \phi_{k}^{(p)}\right\rangle_{L_{p}^{2}(\Sigma)}}
$$

Now (2.11), (2.53)-(2.55) yield (2.15). This completes the proof of Lemma 2.1.

\section{$3 C^{k}$-estimate of the quotient of Bergman kernels}

The proof of Theorem 1.3 follows the same strategy as in Sect. 2 (use of the orthonormal basis $\left(\sigma_{j}^{(p)}\right)_{1 \leq j \leq d_{p}}$ ), but with some play on the parameters (in particular, the truncation floor $\delta_{p}$ of Step 1. in the outline of the proof of Theorem 1.2). Some precisions on this basis are also needed: we'll see more precisely that in some sense, and provided relevant choices along the construction, the head terms $\sigma_{\ell}^{(p)}, 1 \leq \ell \leq \delta_{p}$ are much closer to their counterparts $c_{\ell}^{(p)} z^{\ell}$ of $\mathbb{D}^{*}$ than sketched above.

This section is organized as follows. In Sect. 3.1, we establish a refinement of the integral estimate Lemma 2.1 which is again deduced from [5]. In Sect. 3.2, we establish Theorem 1.3 by using Lemma 3.1 .

\subsection{A refined integral estimate}

To establish Theorem 1.3, we follow Steps 1. to 4. in the outline of the proof of Theorem 1.2 by modifying $\delta_{p}$, thus refining Lemmas 2.1 to 3.1 below.

Let $\kappa>0$ fixed. We start by choosing $c(\kappa) \in\left(0, e^{-1}\right)$ so that

$$
\log (c(\kappa)) \leq-1-2 \kappa .
$$


Then we replace $\delta_{p}$ in (2.10) by

$$
\delta_{p}^{\prime}=\delta_{p}^{\prime}(\kappa)=\left\lfloor\frac{(p-2) c(\kappa)}{2|\log r|}\right\rfloor-2 .
$$

Lemma 3.1 There exists $C=C(\kappa)>0$ such that for all $p \geq 1$ and $\ell \in\left\{1, \ldots, \delta_{p}^{\prime}\right\}$,

$$
\left\|\sigma_{\ell}^{(p)}-c_{\ell}^{(p)} \chi(|z|) z^{\ell} \mathfrak{e}_{L}^{p}\right\|_{L_{p}^{2}(\Sigma)} \leq C p e^{-\kappa p}
$$

Moreover, $\left(\sigma_{\ell}^{(p)}\right)_{1 \leq \ell \leq d_{p}}$ is in echelon form up to rank $\delta_{p}^{\prime}$, in the sense that if $\ell=1, \ldots, \delta_{p}^{\prime}$, then $\sigma_{\ell}^{(p)}$ admits an expansion

$$
\sigma_{\ell}^{(p)}=\left(\sum_{q=\ell}^{\infty} a_{q \ell}^{(p)} z^{q}\right) \mathfrak{e}_{L}^{p} \quad \text { on } \mathbb{D}_{4 r}^{*},
$$

and if $\ell=\delta_{p}^{\prime}+1, \ldots, d_{p}$, then $\sigma_{\ell}^{(p)}$ admits an expansion

$$
\sigma_{\ell}^{(p)}=\left(\sum_{q=\delta_{p}^{\prime}+1}^{\infty} a_{q \ell}^{(p)} z^{q}\right) \mathfrak{e}_{L}^{p} \quad \text { on } \mathbb{D}_{4 r}^{*} .
$$

As will be seen, estimate (3.3) is directly related to the play on $\delta_{p}^{\prime}$, whereas the echelon property as such is not, and (3.4), (3.5) are a direct consequence of (2.30) and (2.31). Moreover, no estimate is given on the $\sigma_{\ell}^{(p)}$ for $\ell \geq \delta_{p}^{\prime}+1$ in the above statement; as in the proof of Theorem 1.2, it turns out that we content ourselves with rather rough estimates on these tail sections.

Proof of Lemma 3.1 Let $\bar{\partial}^{L^{p} *}$ be the formal adjoint of $\bar{\partial}^{L^{p}}$ on $\left(C_{0}^{\infty}\left(\Sigma, L^{p}\right),\|\|_{\boldsymbol{L}_{p}^{2}(\Sigma)}\right)$. Then

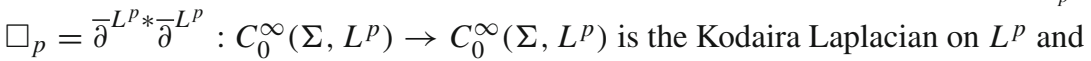

$$
\operatorname{ker} \square_{p}=H_{(2)}^{0}\left(\Sigma, L^{p}\right) \text {. }
$$

Observe that the construction of the $\phi_{\ell}^{(p)}, \ell=1, \ldots, \delta_{p}^{\prime}$, following Steps 1. to 4 . of the proof of Theorem 1.2 can be led alternatively by the following principle:

1 '. with the cut-off function $\chi$ in (2.10), for $\ell=1, \ldots, \delta_{p}^{\prime}$, set

$$
\phi_{0, \ell}^{(p)}:=\phi_{\ell, 0}^{(p)}=c_{\ell}^{(p)} \chi(|z|) z^{\ell} \mathfrak{e}_{L}^{p} .
$$

2'. give an explicit estimate of $\left\|\square_{p} \phi_{0, \ell}^{(p)}\right\|_{\boldsymbol{L}_{p}^{2}(\Sigma)}$.

3'. we correct $\phi_{0, \ell}^{(p)}$ into holomorphic $\boldsymbol{L}^{2}$-section $\phi_{\ell}^{(p)}$ of $L^{p}$, by orthogonal $\boldsymbol{L}_{p}^{2}(\Sigma)$ projection. we use the spectral gap property [5,Corollary 5.2] (as a direct consequence of [22,Theorem 6.1.1]) together with the step 2' to get (3.3).

Step 1'. We compute, by (2.6) and (2.10), as in (2.39), for $\ell=1, \ldots, \delta_{p}^{\prime}$,

$$
\begin{aligned}
0 \leq 1-\left\|c_{\ell}^{(p)} z^{\ell} \chi(|z|) e_{L}^{p}\right\|_{L_{p}^{2}(\Sigma)}^{2} & =\frac{1}{(p-2) !} \int_{0}^{2 \ell \beta|\log r|} u^{p-2} e^{-u}\left(1-\chi^{2}\left(e^{-u /(2 \ell)}\right)\right) d u \\
& \leq \frac{1}{(p-2) !} \int_{0}^{2 \delta_{p}^{\prime} \beta|\log r|} u^{p-2} e^{-u} d u .
\end{aligned}
$$


As $u^{p-2} e^{-u}$ is strictly increasing on $[0, p-2]$ and $\log \beta<0$, and from (3.2), 2( $\delta_{p}^{\prime}+$ 2) $|\log r| \leq(p-2) c(\kappa)$, by (2.28), (3.1), we get a refinement of (2.41),

$$
\begin{aligned}
& \frac{1}{(p-2) !} \int_{0}^{2 \delta_{p}^{\prime} \beta|\log r|} u^{p-2} e^{-u} d u \leq \frac{1}{(p-2) !} \int_{0}^{(p-2) c(\kappa) \beta} u^{p-2} e^{-u} d u \\
& \leq \frac{(p-2)^{p-2}}{(p-2) !} e^{(p-2)(\log (c(\kappa) \beta)-c(\kappa) \beta)}(p-2) c(\kappa) \beta \\
& =\left(\frac{p-2}{2 \pi}\right)^{1 / 2} c(\kappa) \beta\left(1+\mathcal{O}\left(p^{-1}\right)\right) e^{(p-2)(\log (c(\kappa) \beta)-c(\kappa) \beta+1)} \\
& =\mathcal{O}\left(e^{-2 \kappa p}\right) .
\end{aligned}
$$

From (3.8) and (3.9), uniformly in $\ell \in\left\{1, \ldots, \delta_{p}^{\prime}\right\}$,

$$
\left\|\phi_{0, \ell}^{(p)}\right\|_{\boldsymbol{L}_{p}^{2}(\Sigma)}^{2}=\left\|c_{\ell}^{(p)} z^{\ell} \chi(|z|) \mathfrak{e}_{L}^{p}\right\|_{\boldsymbol{L}_{p}^{2}(\Sigma)}^{2}=1+\mathcal{O}\left(e^{-2 \kappa p}\right) .
$$

Step 2'. Recall from [5,(4.13), (4.14), (4.15) or (4.30)] that on $\mathbb{D}_{2 r}^{*}$ (seen in $\Sigma$ ),

$$
\square_{p}\left(\cdot \mathfrak{e}_{L}^{p}\right)=\left(-|z|^{2} \log ^{2}\left(|z|^{2}\right) \frac{\partial^{2} \cdot}{\partial z \partial \bar{z}}-p \bar{z} \log \left(|z|^{2}\right) \frac{\partial \cdot}{\partial \bar{z}}\right) \mathfrak{e}_{L}^{p} .
$$

Hence we obtain from (3.7) and (3.11), for $\ell=1, \ldots, \delta_{p}^{\prime}$,

$$
\square_{p} \phi_{0, \ell}^{(p)}=c_{\ell}^{(p)}\left(-|z|^{2} \log ^{2}\left(|z|^{2}\right) \frac{\partial^{2}}{\partial z \partial \bar{z}}\left(\chi(|z|) z^{\ell}\right)-p \bar{z} \log \left(|z|^{2}\right) \frac{\partial}{\partial \bar{z}}\left(\chi(|z|) z^{\ell}\right)\right) \mathfrak{e}_{L}^{p} .
$$

Since $\frac{\partial}{\partial \bar{z}}\left[\chi(|z|) z^{\ell}\right]=\left(\frac{\partial}{\partial \bar{z}} \chi(|z|)\right) z^{\ell}=\frac{|z|}{2 \bar{z}} \chi^{\prime}(|z|) z^{\ell}$, we have

$$
\frac{\partial^{2}}{\partial z \partial \bar{z}}\left[\chi(|z|) z^{\ell}\right]=\frac{2 \ell+1}{4|z|} z^{\ell} \chi^{\prime}(|z|)+\frac{1}{4} z^{\ell} \chi^{\prime \prime}(|z|),
$$

which yields

$$
\begin{aligned}
& \square_{p} \phi_{0, \ell}^{(p)}=c_{\ell}^{(p)}\left(-\frac{2 \ell+1}{4}|z| z^{\ell} \log ^{2}\left(|z|^{2}\right) \chi^{\prime}(|z|)\right. \\
& \left.-\frac{1}{4}|z|^{2} z^{\ell} \log ^{2}\left(|z|^{2}\right) \chi^{\prime \prime}(|z|)-\frac{p}{2}|z| z^{\ell} \log \left(|z|^{2}\right) \chi^{\prime}(|z|)\right) \mathfrak{e}_{L}^{p}
\end{aligned}
$$

on $\mathbb{D}_{2 r}^{*}$, and this readily extends to the whole $\Sigma$. Therefore,

$$
\begin{aligned}
& \left\|\square_{p} \phi_{0, \ell}^{(p)}\right\|_{L_{p}^{2}(\Sigma)} \leq c_{\ell}^{(p)}\left(\frac{2 \ell+1}{4}\left\||z|^{\ell+1} \log ^{2}\left(|z|^{2}\right) \chi^{\prime}(|z|)\right\|_{L_{p}^{2}\left(\mathbb{D}^{*}\right)}\right. \\
& \left.\quad+\frac{1}{4}\left\||z|^{\ell+2} \log ^{2}\left(|z|^{2}\right) \chi^{\prime \prime}(|z|)\right\|_{L_{p}^{2}\left(\mathbb{D}^{*}\right)}+\frac{p}{2}\left\||z|^{\ell+1} \log \left(|z|^{2}\right) \chi^{\prime}(|z|)\right\|_{L_{p}^{2}\left(\mathbb{D}^{*}\right)}\right) .
\end{aligned}
$$

Using nonetheless arguments similar to those of Step 1'. above, we claim that there exists $C>0$ such that for all $p \geq 1$ and $\ell=1, \ldots, \delta_{p}^{\prime}$,

$$
\begin{aligned}
\left\||z|^{\ell+1} \log ^{2}\left(|z|^{2}\right) \chi^{\prime}(|z|)\right\|_{L_{p}^{2}\left(\mathbb{D}^{*}\right)} & \leq C\left(c_{\ell+1}^{(p+4)}\right)^{-1} e^{-\kappa p}, \\
\left\||z|^{\ell+2} \log ^{2}\left(|z|^{2}\right) \chi^{\prime \prime}(|z|)\right\|_{L_{p}^{2}\left(\mathbb{D}^{*}\right)} & \leq C\left(c_{\ell+2}^{(p+4)}\right)^{-1} e^{-\kappa p}, \\
\left\||z|^{\ell+1} \log \left(|z|^{2}\right) \chi^{\prime}(|z|)\right\|_{L_{p}^{2}\left(\mathbb{D}^{*}\right)} & \leq C\left(c_{\ell+1}^{(p+2)}\right)^{-1} e^{-\kappa p} .
\end{aligned}
$$


Indeed, from (3.2) and since $2\left(\delta_{p}^{\prime}+2\right)|\log r| \leq(p-2) c(\kappa)$, applying (3.9) for $p+4$ as in (2.39), we get with $C_{0}=\sup _{[0,1]}\left|\chi^{\prime}\right|$,

$$
\begin{aligned}
\| c_{\ell+1}^{(p+4)}|z|^{\ell+1} & \log ^{2}\left(|z|^{2}\right) \chi^{\prime}(|z|) \|_{L_{p}^{2}\left(\mathbb{D}^{*}\right)}^{2} \\
& =\left(c_{\ell+1}^{(p+4)}\right)^{2} \int_{r^{\beta}}^{1}\left|\log \left(t^{2}\right)\right|^{p+4} t^{2 \ell+2} \chi^{\prime}(t)^{2} \frac{4 \pi t d t}{t^{2}\left|\log \left(t^{2}\right)\right|^{2}} \\
& u=-2 \underline{\underline{(\ell+1)} \log t} \frac{1}{(p+2) !} \int_{0}^{2(\ell+1) \beta|\log r|} u^{p+2} e^{-u}\left(\chi^{\prime}\left(e^{-\frac{u}{2(\ell+1)}}\right)\right)^{2} d u \\
& \leq \frac{C_{0}^{2}}{(p+2) !} \int_{0}^{2\left(\delta_{p}^{\prime}+1\right) \beta|\log r|} u^{p+2} e^{-u} d u \\
& \leq \frac{C_{0}^{2}}{(p+2) !} \int_{0}^{(p+2) c(\kappa) \beta} u^{p+2} e^{-u} d u=\mathcal{O}\left(e^{-2 \kappa p}\right) .
\end{aligned}
$$

Consequently, by (3.15) and (3.16), there exists $C>0$ such that for all $p \geq 1$ and $\ell=$ $1, \ldots, \delta_{p}^{\prime}$

$$
\begin{aligned}
& \left\|\square_{p} \phi_{0, \ell}^{(p)}\right\|_{L_{p}^{2}} \leq C c_{\ell}^{(p)}\left(\ell\left(c_{\ell+1}^{(p+4)}\right)^{-1}+\left(c_{\ell+2}^{(p+4)}\right)^{-1}+p\left(c_{\ell+1}^{(p+2)}\right)^{-1}\right) e^{-\kappa p} \\
& \quad=C\left(\frac{\ell^{p-1}}{(p-2) !}\right)^{\frac{1}{2}}\left(\ell\left(\frac{(p+2) !}{(\ell+1)^{p+3}}\right)^{\frac{1}{2}}+\left(\frac{(p+2) !}{(\ell+2)^{p+3}}\right)^{\frac{1}{2}}+p\left(\frac{p !}{(\ell+1)^{p+1}}\right)^{\frac{1}{2}}\right) e^{-\kappa p} \\
& \leq C p^{2} e^{-\kappa p} .
\end{aligned}
$$

Step 3'. Recall that the spectral gap property [5,Corollary 5.2] tells us that there exists $C_{1}>0$ such that for all $p \gg 1$ we have

$$
\operatorname{Spec}\left(\square_{p}\right) \subset\{0\} \cup\left[C_{1} p,+\infty\right) .
$$

For $\ell \in\left\{1, \ldots, \delta_{p}^{\prime}\right\}$ let $\psi_{0, \ell}^{(p)} \in \boldsymbol{L}_{p}^{2}(\Sigma)$ such that $\psi_{0, \ell}^{(p)} \perp H_{(2)}^{0}\left(\Sigma, L^{p}\right)$ and $\square_{p} \psi_{0, \ell}^{(p)}=$ $\square_{p} \phi_{0, \ell}^{(p)}$. Then by (3.6),

$$
\phi_{\ell}^{(p)}=\phi_{0, \ell}^{(p)}-\psi_{0, \ell}^{(p)}
$$

By (3.18), (3.19) and (3.20) we get

$$
\left\|\phi_{\ell}^{(p)}-\phi_{0, \ell}^{(p)}\right\|_{L_{p}^{2}(\Sigma)}=\left\|\psi_{0, \ell}^{(p)}\right\|_{L_{p}^{2}(\Sigma)} \leq\left(C_{1} p\right)^{-1}\left\|\square_{p} \phi_{0, \ell}^{(p)}\right\|_{L_{p}^{2}(\Sigma)} \leq C C_{1}^{-1} p e^{-\kappa p},
$$

uniformly in $\ell=1, \ldots, \delta_{p}^{\prime}$. Note that (3.10) can be reformulated as

$$
\left\langle\phi_{0, \ell}^{(p)}, \phi_{0, j}^{(p)}\right\rangle_{L_{p}^{2}(\Sigma)}=\delta_{j \ell}\left(1+\mathcal{O}\left(e^{-2 \kappa p}\right)\right),
$$

(the case $\ell \neq j$ provides 0 by circle symmetry). Thus (3.20)-(3.22) entail

$$
\left\langle\phi_{\ell}^{(p)}, \phi_{j}^{(p)}\right\rangle_{\boldsymbol{L}_{p}^{2}(\Sigma)}=\left\langle\phi_{0, \ell}^{(p)}, \phi_{0, j}^{(p)}\right\rangle_{\boldsymbol{L}_{p}^{2}(\Sigma)}-\left\langle\psi_{0, \ell}^{(p)}, \psi_{0, j}^{(p)}\right\rangle_{\boldsymbol{L}_{p}^{2}(\Sigma)}=\delta_{j \ell}+\mathcal{O}\left(p^{2} e^{-2 \kappa p}\right),
$$

uniformly in $\ell, j=1, \ldots, \delta_{p}^{\prime}$. Because $\left(\sigma_{\ell}^{(p)}\right)_{1 \leq \ell \leq \delta_{p}^{\prime}}$ is obtained by the Gram-Schmidt orthonormalisation of $\left(\phi_{\ell}^{(p)}\right)_{1 \leq \ell \leq \delta_{p}^{\prime}}$ (which is a $\delta_{p}^{\prime}=\mathcal{O}(p)$ process) we infer from (2.55) and (3.23) that

$$
\left\|\sigma_{\ell}^{\prime(p)}-\phi_{\ell}^{(p)}\right\|_{L_{p}^{2}(\Sigma)}=\mathcal{O}\left(p^{3} e^{-2 \kappa p}\right), \quad\left\|\sigma_{\ell}^{\prime(p)}\right\|_{L_{p}^{2}(\Sigma)}=1+\mathcal{O}\left(p^{3} e^{-2 \kappa p}\right) .
$$


Since $\sigma_{\ell}^{(p)}=\sigma_{\ell}^{\prime(p)} /\left\|\sigma_{\ell}^{\prime(p)}\right\|_{\boldsymbol{L}_{p}^{2}(\Sigma)}$, we conclude from (3.24) that there exists $C>0$ such that for $p \gg 1$,

$$
\left\|\sigma_{\ell}^{(p)}-\phi_{\ell}^{(p)}\right\|_{\boldsymbol{L}_{p}^{2}(\Sigma)} \leq\left|\left\|\sigma_{\ell}^{\prime(p)}\right\|_{\boldsymbol{L}_{p}^{2}(\Sigma)}-1\right|+\left\|\sigma_{\ell}^{\prime(p)}-\phi_{\ell}^{(p)}\right\|_{\boldsymbol{L}_{p}^{2}(\Sigma)} \leq C p^{3} e^{-2 \kappa p}
$$

hence, by (3.21) and (3.25) that we have uniformly in $\ell=1, \ldots, \delta_{p}^{\prime}$ for $p \gg 1$,

$$
\left\|\sigma_{\ell}^{(p)}-c_{\ell}^{(p)} \chi(|z|) z^{\ell} \mathfrak{e}_{L}^{p}\right\|_{L_{p}^{2}(\Sigma)}=\left\|\sigma_{\ell}^{(p)}-\phi_{0, \ell}^{(p)}\right\|_{L_{p}^{2}(\Sigma)} \leq C p e^{-\kappa p} .
$$

Echelon property. - We use the expansion (2.22) of $\sigma_{\ell}^{p}$ on $\mathbb{D}_{4 r}^{*}$. By construction, $\phi_{j}^{(p)} \in$ $\operatorname{Span}\left\{\sigma_{1}^{(p)}, \ldots, \sigma_{j}^{(p)}\right\}$ for $1 \leq j \leq \delta_{p}^{\prime}$, so if $j<\ell$, then $\phi_{j}^{(p)} \perp_{L_{p}^{2}(\Sigma)} \sigma_{\ell}^{(p)}$. As $\phi_{j}^{(p)}$ is the $\boldsymbol{L}_{p}^{2}(\Sigma)$-projection of $\phi_{0, j}^{(p)}$ on holomorphic sections, we have as in (2.31) that

$$
\left\langle\sigma_{\ell}^{(p)}, \phi_{0, j}^{(p)}\right\rangle_{L_{p}^{2}(\Sigma)}=\left\langle\sigma_{\ell}^{(p)}, \phi_{j}^{(p)}\right\rangle_{L_{p}^{2}(\Sigma)}=0, \quad \text { if } j<\ell
$$

Now (2.30) and (3.27) entail

$$
a_{j \ell}^{(p)}=0 \quad \text { if } j<\ell, j \in\left\{1, \ldots, \delta_{p}^{\prime}\right\}, \ell \in\left\{1, \ldots, d_{p}\right\} .
$$

From (2.22) and (3.28) we get (3.4) and (3.5). The proof of Lemma 3.1 is completed.

The following consequence of Lemma 3.1 that refines (2.34) is very useful in our computations.

Lemma 3.2 We have uniformly for $j, \ell \in\left\{1, \ldots, \delta_{p}^{\prime}\right\}$,

$$
a_{j \ell}^{(p)}=\left\{\begin{array}{lr}
0 & \text { for } j<\ell ; \\
c_{j}^{(p)}\left(\delta_{j \ell}+\mathcal{O}\left(p e^{-\kappa p}\right)\right) & \text { for } j \geq \ell .
\end{array}\right.
$$

Proof First note that by (3.20) we have

$$
\left\langle\sigma_{\ell}^{(p)}, \phi_{j}^{(p)}\right\rangle_{\boldsymbol{L}_{p}^{2}(\Sigma)}=\left\langle\sigma_{\ell}^{(p)}, \phi_{0, j}^{(p)}\right\rangle_{\boldsymbol{L}_{p}^{2}(\Sigma)}=\left\langle\sigma_{\ell}^{(p)}-\phi_{0, \ell}^{(p)}, \phi_{0, j}^{(p)}\right\rangle_{\boldsymbol{L}_{p}^{2}(\Sigma)}+\left\langle\phi_{0, \ell}^{(p)}, \phi_{0, j}^{(p)}\right\rangle_{\boldsymbol{L}_{p}^{2}(\Sigma)}
$$

Further, (3.3), (3.22) and (3.30) imply

$$
\left\langle\sigma_{\ell}^{(p)}, \phi_{j}^{(p)}\right\rangle_{\boldsymbol{L}_{p}^{2}(\Sigma)}=\mathcal{O}\left(p e^{-\kappa p}\right)+\delta_{j \ell}\left(1+\mathcal{O}\left(e^{-2 \kappa p}\right)\right)
$$

By (2.38) and (3.10) we have uniformly on $j \in\left\{1, \ldots, \delta_{p}^{\prime}\right\}$,

$$
\left(c_{j}^{(p)}\right)^{2} \int_{\mathbb{D}_{2 r}^{*}}\left|\log \left(|z|^{2}\right)\right|^{p}|z|^{2 j} \chi(|z|) \omega_{\mathbb{D}^{*}}=1+\mathcal{O}\left(e^{-2 \kappa p}\right) .
$$

The first equality of (2.34), (3.28), (3.31) and (3.32) entail (3.29).

\subsection{Proof of Theorem 1.3}

We show now how to establish Theorem 1.3 by using Lemma 3.1. It can be noticed here that while estimate (3.3) is essential in establishing Theorem 1.3, the echelon property is not, but helps nonetheless clarify some of the upcoming computations.

The proof goes as follows: we start by explicit computations, then use Lemma 3.1 to lead a precise analysis of head terms, i.e., all its indices $\leq \delta_{p}^{\prime}$; and recall some rough estimates of tail terms, i.e., some of its indices $\geq \delta_{p}^{\prime}+1$. 
On some shrinking disc family $\left\{|z| \leq c^{\prime} p^{-A^{\prime}}\right\}$, we will conclude from (2.23) that the tails terms can be controlled by $2^{-\alpha^{\prime} p} B_{p}^{\mathbb{D}^{*}}$, hence on some fixed trivialization disc, as for Theorem 1.2.

From (2.42), for $z \in \mathbb{D}_{4 r}^{*}$, set

$$
\beta_{p}^{\Sigma}(z)=\beta_{p}^{\Sigma}(z, z), \quad \beta_{p}^{\mathbb{D}^{*}}(z)=\beta_{p}^{\mathbb{D}^{*}}(z, z) .
$$

By (2.42) and (3.33), we have

$$
\frac{B_{p}(z)}{B_{p}^{\mathbb{D}^{*}}(z)}=\frac{\beta_{p}^{\Sigma}(z)}{\beta_{p}^{\mathbb{D}^{*}}(z)}=1+\left(\beta_{p}^{\Sigma}-\beta_{p}^{\mathbb{D}^{*}}\right)(z)\left(\beta_{p}^{\mathbb{D}^{*}}(z)\right)^{-1} .
$$

With the notations of Lemma 3.1 we compute explicitly on $\mathbb{D}_{4 r}^{*}$. By (1.4), (2.7), (2.22), (2.42) and (3.33), we have

$$
\beta_{p}^{\Sigma}(z)=\sum_{q, s=1}^{\infty}\left(\sum_{\ell=1}^{d_{p}} a_{q \ell}^{(p)} \overline{a_{s \ell}^{(p)}}\right) z^{q} \bar{z}^{s}, \quad \beta_{p}^{\mathbb{D}^{*}}(z)=\sum_{q=1}^{\infty}\left(c_{q}^{(p)}\right)^{2}|z|^{2 q} .
$$

For $q, s \in \mathbb{N}^{*}$, set

$$
\epsilon_{q s}=\sum_{\ell=1}^{d_{p}} \frac{a_{q \ell}^{(p)}}{c_{q}^{(p)}} \frac{\overline{a_{s \ell}^{(p)}}}{c_{s}^{(p)}}-\delta_{q s} .
$$

From (3.35) and (3.36), we get

$$
\begin{aligned}
& \frac{d}{d z}\left(\beta_{p}^{\Sigma}-\beta_{p}^{\mathbb{D}^{*}}\right)(z) \cdot \beta_{p}^{\mathbb{D}^{*}}(z) \\
& \quad=\sum_{q, s=1}^{\infty} q\left(\sum_{\ell=1}^{d_{p}} a_{q \ell}^{(p)} \overline{a_{s \ell}^{(p)}} z^{q-1} \bar{z}^{s}-\delta_{q s}\left(c_{q}^{(p)}\right)^{2} z^{q-1} \bar{z}^{s}\right) \cdot \sum_{m=1}^{\infty}\left(c_{m}^{(p)}\right)^{2}|z|^{2 m} \\
& \quad=\sum_{q, s, m=1}^{\infty} q\left(c_{m}^{(p)}\right)^{2} c_{q}^{(p)} c_{s}^{(p)} \epsilon_{q s} z^{q+m-1} \bar{z}^{s+m},
\end{aligned}
$$

and similarly,

$$
\left(\beta_{p}^{\Sigma}-\beta_{p}^{\mathbb{D}^{*}}\right)(z) \cdot \frac{d}{d z} \beta_{p}^{\mathbb{D}^{*}}(z)=\sum_{q, s, m=1}^{\infty} m\left(c_{m}^{(p)}\right)^{2} c_{q}^{(p)} c_{s}^{(p)} \epsilon_{q s} z^{q+m-1} \bar{z}^{s+m} .
$$

From (3.34), (3.37) and (3.38), we get

$$
\frac{d}{d z} \frac{B_{p}}{B_{p}^{\mathbb{D}^{*}}}(z)=\left(\beta_{p}^{\mathbb{D}^{*}}(z)\right)^{-2} \sum_{q, s, m=1}^{\infty}\left[(q-m)\left(c_{m}^{(p)}\right)^{2} c_{q}^{(p)} c_{s}^{(p)} \epsilon_{q s}\right] z^{q+m-1} \bar{z}^{s+m} .
$$

Observe that the coefficient inside [...] in the above sum vanishes if $q=m$. This allows to separate the above sum into

$$
\sum_{q=1, s \geq 1, m \geq 2} \text { and } \sum_{q \geq 2, s \geq 1, m \geq 1} .
$$

We first tackle the sum over $q=1, s \geq 1$ and $m \geq 2$, focusing on the cases $s, m \leq \delta_{p}^{\prime}$; then we deal with the sum over $q \geq 2, s \geq 1$ and $m \geq 1$, focusing on $q, s, m \leq \delta_{p}^{\prime}$, before we also address the cases of "large indices" $\left(\max \{q, s, m\} \geq \delta_{p}^{\prime}+1\right)$. 
Head terms. - We look at first

$$
I_{p, \delta_{p}^{\prime}}(z)=\sum_{s=1}^{\delta_{p}^{\prime}} \sum_{m=2}^{\delta_{p}^{\prime}}\left[(1-m)\left(c_{m}^{(p)}\right)^{2} c_{1}^{(p)} c_{s}^{(p)} \epsilon_{1 s}\right] z^{m} \bar{z}^{s+m} .
$$

By (3.4), (3.5), (3.29) and (3.36), uniformly for $q, s \in\left\{1, \ldots, \delta_{p}^{\prime}\right\}$,

$$
\epsilon_{q s}=\sum_{\ell=1}^{\min \{q, s\}} \frac{a_{q \ell}^{(p)}}{c_{q}^{(p)}} \frac{\overline{a_{s \ell}^{(p)}}}{c_{s}^{(p)}}-\delta_{q s}=\mathcal{O}\left(\delta_{p}^{\prime} p e^{-\kappa p}\right) .
$$

For all $t \in\left\{2, \ldots, \delta_{p}^{\prime}\right\}, j \in\left\{1, \ldots, \delta_{p}^{\prime}\right\}$, by (2.6),

$$
\left|(t-j) c_{t}^{(p)}\right| \leq \delta_{p}^{\prime}\left(\frac{t}{t-1}\right)^{(p-1) / 2} c_{t-1}^{(p)} \leq \delta_{p}^{\prime} 2^{(p-1) / 2} c_{t-1}^{(p)}
$$

From (3.40)-(3.42), we get

$$
\begin{aligned}
\left|I_{p, \delta_{p}^{\prime}}(z)\right| & \leq \sum_{s=1}^{\delta_{p}^{\prime}} \sum_{m=2}^{\delta_{p}^{\prime}}(m-1)\left(c_{m}^{(p)}\right)^{2} c_{1}^{(p)} c_{s}^{(p)}\left|\epsilon_{1 s}\right||z|^{s+2 m} \\
& \leq \mathcal{O}\left(\left(\delta_{p}^{\prime}\right)^{2} 2^{p / 2} p e^{-\kappa p}\right) \cdot \sum_{s=1}^{\delta_{p}^{\prime}} \sum_{m=2}^{\delta_{p}^{\prime}} c_{m}^{(p)} c_{m-1}^{(p)} c_{1}^{(p)} c_{s}^{(p)}|z|^{s+2 m} .
\end{aligned}
$$

But

$$
\begin{aligned}
& \sum_{s=1}^{\delta_{p}^{\prime}} \sum_{m=2}^{\delta_{p}^{\prime}} c_{m}^{(p)} c_{m-1}^{(p)} c_{1}^{(p)} c_{s}^{(p)}|z|^{s+2 m}=\left(\sum_{s=1}^{\delta_{p}^{\prime}} c_{1}^{(p)} c_{s}^{(p)}|z|^{1+s}\right)\left(\sum_{m=2}^{\delta_{p}^{\prime}} c_{m}^{(p)} c_{m-1}^{(p)}|z|^{2 m-1}\right) \\
& \leq \frac{1}{2}\left(\delta_{p}^{\prime}\left(c_{1}^{(p)}\right)^{2}|z|^{2}+\sum_{s=1}^{\delta_{p}^{\prime}}\left(c_{s}^{(p)}\right)^{2}|z|^{2 s}\right)\left(\sum_{m=2}^{\delta_{p}^{\prime}}\left(c_{m}^{(p)}\right)^{2}|z|^{2 m}\right)^{\frac{1}{2}}\left(\sum_{m=2}^{\delta_{p}^{\prime}}\left(c_{m-1}^{(p)}\right)^{2}|z|^{2(m-1)}\right)^{\frac{1}{2}} \\
& \leq\left(\delta_{p}^{\prime}+1\right)\left(\sum_{j=1}^{\infty}\left(c_{j}^{(p)}\right)^{2}|z|^{2 j}\right)^{2}=\left(\delta_{p}^{\prime}+1\right)\left(\beta_{p}^{\mathbb{D}^{*}}(z)\right)^{2} .
\end{aligned}
$$

We proceed similarly with the sum

$$
I I_{p, \delta_{p}^{\prime}}(z)=\sum_{q=2}^{\delta_{p}^{\prime}} \sum_{s=1}^{\delta_{p}^{\prime}} \sum_{m=1}^{\delta_{p}^{\prime}}\left[(q-m)\left(c_{m}^{(p)}\right)^{2} c_{q}^{(p)} c_{s}^{(p)} \epsilon_{q s}\right] z^{q+m-1} \bar{z}^{s+m}
$$


We have analogously to (3.44),

$$
\begin{aligned}
& \sum_{q=2}^{\delta_{p}^{\prime}} \sum_{s=1}^{\delta_{p}^{\prime}} \sum_{m=1}^{\delta_{p}^{\prime}}\left(c_{m}^{(p)}\right)^{2} c_{q-1}^{(p)} c_{s}^{(p)}|z|^{q+s+2 m-1} \\
& \quad=\left(\sum_{q=2}^{\delta_{p}^{\prime}} c_{q-1}^{(p)}|z|^{q-1}\right)\left(\sum_{s=1}^{\delta_{p}^{\prime}} c_{s}^{(p)}|z|^{s}\right)\left(\sum_{m=1}^{\delta_{p}^{\prime}}\left(c_{m}^{(p)}\right)^{2}|z|^{2 m}\right) \\
& \quad \leq \delta_{p}^{\prime}\left(\sum_{q=2}^{\delta_{p}^{\prime}}\left(c_{q-1}^{(p)}\right)^{2}|z|^{2 q-2}\right)^{\frac{1}{2}}\left(\sum_{s=1}^{\delta_{p}^{\prime}}\left(c_{s}^{(p)}\right)^{2}|z|^{2 s}\right)^{\frac{1}{2}}\left(\sum_{m=1}^{\delta_{p}^{\prime}}\left(c_{m}^{(p)}\right)^{2}|z|^{2 m}\right) \\
& \quad \leq \delta_{p}^{\prime}\left(\beta_{p}^{\mathbb{D}^{*}}(z)\right)^{2} .
\end{aligned}
$$

From (3.41), (3.42), (3.45) and (3.46), we get

$$
\begin{aligned}
\left|I I_{p, \delta_{p}^{\prime}}(z)\right| & \leq \sum_{q=2}^{\delta_{p}^{\prime}} \sum_{s=1}^{\delta_{p}^{\prime}} \sum_{m=1}^{\delta_{p}^{\prime}}\left(c_{m}^{(p)}\right)^{2}\left|(q-m) c_{q}^{(p)}\right| c_{s}^{(p)}\left|\epsilon_{q s}\right||z|^{q+s+2 m-1} \\
& \leq \mathcal{O}\left(\left(\delta_{p}^{\prime}\right)^{2} 2^{p / 2} p e^{-\kappa p}\right) \cdot \sum_{q=2}^{\delta_{p}^{\prime}} \sum_{s=1}^{\delta_{p}^{\prime}} \sum_{m=1}^{\delta_{p}^{\prime}}\left(c_{m}^{(p)}\right)^{2} c_{q-1}^{(p)} c_{s}^{(p)}|z|^{q+s+2 m-1} \\
& \leq \mathcal{O}\left(\left(\delta_{p}^{\prime}\right)^{3} 2^{p / 2} p e^{-\kappa p}\right) \cdot\left(\beta_{p}^{\mathbb{D}^{*}}(z)\right)^{2}
\end{aligned}
$$

Tail terms. - Set

$$
\begin{aligned}
& \mathcal{A}_{p}^{1}=\left\{(q, s, m) \in\left(\mathbb{N}^{*}\right)^{3}: q \geq \delta_{p}^{\prime}+1 ; s, m \leq \delta_{p}^{\prime}\right\}, \\
& \mathcal{A}_{p}^{2}=\left\{(q, s, m) \in\left(\mathbb{N}^{*}\right)^{3}: s \geq \delta_{p}^{\prime}+1 ; m \leq \delta_{p}^{\prime}\right\}, \\
& \left.\mathcal{A}_{p}^{3}=\left\{(q, s, m) \in\left(\mathbb{N}^{*}\right)^{3}: m \geq \delta_{p}^{\prime}+1\right\}\right\} .
\end{aligned}
$$

For $j=1,2,3$, set

$$
I\left(\mathcal{A}_{p}^{j}\right)(z)=\sum_{(q, s, m) \in \mathcal{A}_{p}^{j}}(q-m)\left(c_{m}^{(p)}\right)^{2} c_{q}^{(p)} c_{s}^{(p)} \epsilon_{q s} z^{q+m-1} \bar{z}^{s+m} .
$$

By (3.39), (3.40), (3.45) and (3.49), we have

$$
\frac{d}{d z} \frac{B_{p}}{B_{p}^{\mathbb{D}^{*}}}(z)=\left(\beta_{p}^{\mathbb{D}^{*}}(z)\right)^{-2}\left(I_{p, \delta_{p}^{\prime}}(z)+I I_{p, \delta_{p}^{\prime}}(z)+I\left(\mathcal{A}_{p}^{1}\right)(z)+I\left(\mathcal{A}_{p}^{2}\right)(z)+I\left(\mathcal{A}_{p}^{3}\right)(z)\right) .
$$

We now look at the remaining terms of the sum in (3.50), i.e., $I\left(\mathcal{A}_{p}^{j}\right)(j=1,2,3)$.

First, for all triple $(q, s, m)$ of $\mathcal{A}_{p}^{1}$, as $q \geq \delta_{p}^{\prime}+1>\delta_{p}^{\prime} \geq s$, by (3.28), (3.36), one has:

$$
c_{q}^{(p)} c_{s}^{(p)} \epsilon_{q s}=\sum_{\ell=1}^{d_{p}} a_{q \ell}^{(p)} \overline{a_{s \ell}^{(p)}}=\sum_{\ell=1}^{\delta_{p}^{\prime}} a_{q \ell}^{(p)} \overline{a_{s \ell}^{(p)}} .
$$


From (3.49) and (3.51), we have

$$
\begin{aligned}
& \left|I\left(\mathcal{A}_{p}^{1}\right)(z)\right| \leq C d_{p} \sum_{(q, s, m) \in \mathcal{A}_{p}^{1}} q\left(\sup _{1 \leq \ell \leq d_{p}}\left|a_{q \ell}^{(p)}\right|\right)\left(\sup _{1 \leq \ell \leq d_{p}}\left|a_{s \ell}^{(p)}\right|\right)\left(c_{m}^{(p)}\right)^{2}|z|^{q+s+2 m-1} \\
& =C d_{p}\left(\sum_{q=\delta_{p}^{\prime}+1}^{\infty} q\left(\sup _{1 \leq \ell \leq d_{p}}\left|a_{q \ell}^{(p)}\right|\right)|z|^{q-1}\right) \\
& \quad \times\left(\sum_{s=1}^{\delta_{p}^{\prime}}\left(\sup _{1 \leq \ell \leq d_{p}}\left|a_{s \ell}^{(p)}\right|\right)|z|^{s}\right)\left(\sum_{m=1}^{\delta_{p}^{\prime}}\left(c_{m}^{(p)}\right)^{2}|z|^{2 m}\right) .
\end{aligned}
$$

By (3.28) and (3.29) we get uniformly in $j \in\left\{1, \cdots, \delta_{p}^{\prime}\right\}$,

$$
\sup _{1 \leq \ell \leq d_{p}}\left|a_{j \ell}^{(p)}\right|=\sup _{1 \leq \ell \leq \delta_{p}^{\prime}}\left|a_{j \ell}^{(p)}\right| \leq C c_{j}^{(p)} .
$$

By (3.35) and (3.53), observe that for $z \in \mathbb{D}_{r}^{*}$,

$$
\begin{aligned}
& \sum_{s=1}^{\delta_{p}^{\prime}}\left(\sup _{1 \leq \ell \leq d_{p}}\left|a_{s \ell}^{(p)}\right|\right)|z|^{s} \leq C \sum_{s=1}^{\delta_{p}^{\prime}} c_{s}^{(p)}|z|^{s} \\
& \quad \leq C\left(\delta_{p}^{\prime}\right)^{1 / 2}\left(\sum_{s=1}^{\delta_{p}^{\prime}}\left(c_{s}^{(p)}\right)^{2}|z|^{2 s}\right)^{1 / 2} \leq C\left(\delta_{p}^{\prime}\right)^{1 / 2}\left(\beta_{p}^{\mathbb{D}^{*}}(z)\right)^{1 / 2} .
\end{aligned}
$$

Now we give an estimate via $\beta_{p}^{\mathbb{D}^{*}}(z)$ for the sum $\sum_{q \geq \delta_{p}^{\prime}+1}$ in (3.52). Recall that for $\xi \in[0,1)$ and $N \geq 0$ we have that

$$
\sum_{q=N+1}^{\infty} q \xi^{q-1}=\left(\sum_{q=N+1}^{\infty} \xi^{q}\right)^{\prime}=\frac{(N+1) \xi^{N}-N \xi^{N+1}}{(1-\xi)^{2}} \leq \frac{(N+1) \xi^{N}}{(1-\xi)^{2}}
$$

thus, if $|z| \leq r$,

$$
\sum_{q=\delta_{p}^{\prime}+1}^{\infty} q\left(\frac{|z|}{2 r}\right)^{q-1} \leq\left(\delta_{p}^{\prime}+1\right)\left(\frac{|z|}{2 r}\right)^{\delta_{p}^{\prime}}\left(1-\frac{|z|}{2 r}\right)^{-2} \leq 4\left(\delta_{p}^{\prime}+1\right)\left(\frac{|z|}{2 r}\right)^{\delta_{p}^{\prime}} .
$$

Taking now

$$
A^{\prime}=\frac{1}{2 \alpha^{\prime}}, \quad \alpha^{\prime}=\frac{c(\kappa)}{4|\log r|} \quad \text { and } \quad c^{\prime}=r e^{1 / 2 \alpha^{\prime}}\left|\log \left(|2 r|^{2}\right)\right|^{1 / 2 \alpha^{\prime}},
$$

we obtain from (3.2) that for any $\tau \in \mathbb{N}$ fixed,

$$
\alpha^{\prime} p \leq \delta_{p}^{\prime}-\tau \text { for } \quad p \gg 1 .
$$

Thus, as in (2.27), we have by (3.57) and (3.58) for $\tau \in \mathbb{N}$ fixed,

$$
\left(\frac{|z|}{2 r}\right)^{2\left(\delta_{p}^{\prime}-\tau\right) / p} \frac{1}{\left|\log \left(|2 r|^{2}\right)\right|} \leq\left(\frac{|z|}{2 r}\right)^{2 \alpha^{\prime}} \frac{1}{\left|\log \left(|2 r|^{2}\right)\right|} \leq 2^{-2 \alpha^{\prime}} \frac{e}{p},
$$


for $p \gg 1,|z| \leq c^{\prime} p^{-A^{\prime}}$. To conclude, we estimate by (2.28), (3.35) and (3.59) for any $\tau \in \mathbb{N}$ fixed,

$$
\begin{aligned}
& \left|\log \left(|2 r|^{2}\right)\right|^{-p / 2}\left(\frac{|z|}{2 r}\right)^{\delta_{p}^{\prime}-\tau+1} \\
& \quad=\frac{1}{2 r}\left(\left(\frac{|z|}{2 r}\right)^{2\left(\delta_{p}^{\prime}-\tau\right) / p} \frac{1}{\left|\log \left(|2 r|^{2}\right)\right|}\right)^{p / 2}(2 \pi(p-2) !)^{1 / 2} c_{1}^{(p)}|z| \\
& \quad \leq C p^{-1 / 2} 2^{-\alpha^{\prime} p} \beta_{p}^{\mathbb{D}^{*}}(z)^{1 / 2} .
\end{aligned}
$$

for all $p \gg 1$ and $|z| \leq c^{\prime} p^{-A^{\prime}}$. Thus by (2.23), (3.56) and (3.60) for $\tau=1$, we have for all $p \gg 1$ and $|z| \leq c^{\prime} p^{-A^{\prime}}$,

$$
\begin{aligned}
& \sum_{q=\delta_{p}^{\prime}+1}^{\infty} q\left(\sup _{1 \leq \ell \leq d_{p}}\left|a_{q \ell}^{(p)}\right|\right)|z|^{q-1} \leq \frac{C p^{1 / 2}}{2 r}\left|\log \left(|2 r|^{2}\right)\right|^{-p / 2} \sum_{q=\delta_{p}^{\prime}+1}^{\infty} q\left(\frac{|z|}{2 r}\right)^{q-1} \\
& \leq C \delta_{p}^{\prime} 2^{-\alpha^{\prime} p} \beta_{p}^{\mathbb{D}^{*}}(z)^{1 / 2}
\end{aligned}
$$

By (2.3), (3.52), (3.54) and (3.61) we have for all $p \gg 1$ and $|z| \leq c^{\prime} p^{-A^{\prime}}$,

$$
\left|I\left(\mathcal{A}_{p}^{1}\right)(z)\right| \leq C\left(\delta_{p}^{\prime}\right)^{3 / 2} d_{p} 2^{-\alpha^{\prime} p}\left(\beta_{p}^{\mathbb{D}^{*}}(z)\right)^{2} \leq C p^{5 / 2} 2^{-\alpha^{\prime} p}\left(\beta_{p}^{\mathbb{D}^{*}}(z)\right)^{2}
$$

Sums over $\mathcal{A}_{p}^{2}$ and $\mathcal{A}_{p}^{3}$. - We continue to work on the estimates of the tail terms. We first deal with the sum over $\mathcal{A}_{p}^{2}$. By (3.36) and (3.49), one has:

$$
\begin{aligned}
I\left(\mathcal{A}_{p}^{2}\right)(z)= & \sum_{(q, s, m) \in \mathcal{A}_{p}^{2}}(q-m)\left(c_{m}^{(p)}\right)^{2}\left(\sum_{\ell=1}^{d_{p}} a_{q \ell}^{(p)} \overline{a_{s \ell}^{(p)}}\right) z^{q+m-1} \bar{z}^{s+m} \\
& -\sum_{s=\delta_{p}^{\prime}+1}^{\infty} \sum_{m=1}^{\delta_{p}^{\prime}}(s-m)\left(c_{m}^{(p)}\right)^{2}\left(c_{s}^{(p)}\right)^{2} z^{s+m-1} \bar{z}^{s+m} . \\
= & : S_{1}-S_{2} .
\end{aligned}
$$

Now, since $|q-m| \leq q m$ for all $(q, s, m) \in \mathcal{A}_{p}^{2}$, we obtain,

$$
\left|S_{1}\right| \leq d_{p} \sum_{q=1}^{\infty} q\left(\sup _{1 \leq \ell \leq d_{p}}\left|a_{q \ell}^{(p)}\right|\right)|z|^{q} \cdot \sum_{s=\delta_{p}^{\prime}+1}^{\infty}\left(\sup _{1 \leq \ell \leq d_{p}}\left|a_{s \ell}^{(p)}\right|\right)|z|^{s-1} \cdot \sum_{m=1}^{\delta_{p}^{\prime}} m\left(c_{m}^{(p)}\right)^{2}|z|^{2 m}
$$

By (3.53), (3.54) and (3.61) we get on $|z| \leq c^{\prime} p^{-A^{\prime}}$,

$$
\begin{aligned}
\sum_{q=1}^{\infty} q\left(\sup _{1 \leq \ell \leq d_{p}}\left|a_{q \ell}^{(p)}\right|\right)|z|^{q} & \leq C \delta_{p}^{\prime} \sum_{q=1}^{\delta_{p}^{\prime}} c_{q}^{(p)}|z|^{q}+C|z| \delta_{p}^{\prime} 2^{-\alpha^{\prime} p} \beta_{p}^{\mathbb{D}^{*}}(z)^{1 / 2} \\
& =\mathcal{O}\left(\left(\delta_{p}^{\prime}\right)^{3 / 2}+\delta_{p}^{\prime} 2^{-\alpha^{\prime} p}\right)\left(\beta_{p}^{\mathbb{D}^{*}}(z)\right)^{1 / 2}
\end{aligned}
$$


From (2.23) and (3.60) for $\tau=1$ we infer that we have for $|z| \leq c^{\prime} p^{-A^{\prime}}$,

$$
\begin{aligned}
& \sum_{s=\delta_{p}^{\prime}+1}^{\infty}\left(\sup _{1 \leq \ell \leq d_{p}}\left|a_{s \ell}^{(p)}\right|\right)|z|^{s-1} \leq C p^{1 / 2}\left|\log \left(|2 r|^{2}\right)\right|^{-p / 2} \sum_{s=\delta_{p}^{\prime}+1}^{\infty}\left(\frac{1}{2 r}\right)^{s}|z|^{s-1} \\
& =\frac{C}{2 r} C p^{1 / 2}\left|\log \left(|2 r|^{2}\right)\right|^{-p / 2}\left(\frac{|z|}{2 r}\right)^{\delta_{p}^{\prime}} \frac{1}{1-|z| / 2 r} \\
& \leq C 2^{-\alpha^{\prime} p}\left(\beta_{p}^{\mathbb{D}^{*}}(z)\right)^{1 / 2}
\end{aligned}
$$

Obviously,

$$
\sum_{m=1}^{\delta_{p}^{\prime}} m\left(c_{m}^{(p)}\right)^{2}|z|^{2 m} \leq \delta_{p}^{\prime} \sum_{m=1}^{\delta_{p}^{\prime}}\left(c_{m}^{(p)}\right)^{2}|z|^{2 m} \leq \delta_{p}^{\prime} \beta_{p}^{\mathbb{D}^{*}}(z)
$$

Thus, using these three estimates (3.65)-(3.67) together with (2.3), (3.2), we see that (3.64) yields:

$$
\left|S_{1}\right|=\mathcal{O}\left(p \cdot p^{3 / 2} \cdot p\right) 2^{-\alpha^{\prime} p} \beta_{p}^{\mathbb{D}^{*}}(z)^{2} \text { for }|z| \leq c^{\prime} p^{-A^{\prime}}
$$

From (3.63),

$$
\begin{aligned}
\left|S_{2}\right| & \leq \sum_{s=\delta_{p}^{\prime}+1}^{\infty} \sum_{m=1}^{\delta_{p}^{\prime}}|s-m|\left(c_{m}^{(p)}\right)^{2}\left(c_{s}^{(p)}\right)^{2}|z|^{2 s+2 m-1} \\
& \leq\left(\sum_{s=\delta_{p}^{\prime}+1}^{\infty} s\left(c_{s}^{(p)}\right)^{2}|z|^{2 s-1}\right)\left(\sum_{m=1}^{\delta_{p}^{\prime}}\left(c_{m}^{(p)}\right)^{2}|z|^{2 m}\right) .
\end{aligned}
$$

Note that by the argument in (2.23) for $\mathbb{D}^{*}$ (or directly from $(2.7),(2.8)$ ), there exists $C>0$ such that for any $s \in \mathbb{N}^{*}, p \geq 2$, we have

$$
\left|c_{s}^{(p)}\right| \leq C p^{1 / 2}(2 r)^{-s}\left|\log \left(|2 r|^{2}\right)\right|^{-p / 2} .
$$

By (3.2), (3.55), (3.60) for $\tau=1$, and (3.70), we get as in (3.56) for all $p \gg 1$ and $|z| \leq c^{\prime} p^{-A^{\prime}}$

$$
\begin{aligned}
& \left(\sum_{s=\delta_{p}^{\prime}+1}^{\infty} s\left(c_{s}^{(p)}\right)^{2}|z|^{2 s-1}\right) \leq C\left|\log \left(|2 r|^{2}\right)\right|^{-p} \frac{|z|}{4 r^{2}} p\left(\sum_{s=\delta_{p}^{\prime}+1}^{\infty} s\left(\frac{|z|}{2 r}\right)^{2 s-2}\right) \\
& \leq C p\left|\log \left(|2 r|^{2}\right)\right|^{-p}|z| \frac{\left(\delta_{p}^{\prime}+1\right)}{\left(1-(|z| / 2 r)^{2}\right)^{2}}\left(\frac{|z|}{2 r}\right)^{2 \delta_{p}^{\prime}} \leq C p 2^{-2 \alpha^{\prime} p} \beta_{p}^{\mathbb{D}^{*}}(z)
\end{aligned}
$$

By (3.63), (3.68), (3.69) and (3.71) we obtain

$$
\left|I\left(\mathcal{A}_{p}^{2}\right)(z)\right|=\mathcal{O}\left(p^{7 / 2} 2^{-\alpha^{\prime} p}\right) \beta_{p}^{\mathbb{D}^{*}}(z)^{2} \quad \text { on }|z| \leq c^{\prime} p^{-A^{\prime}} .
$$


We finally deal with the sum over $\mathcal{A}_{p}^{3}$, using the same principles ${ }^{1}$. Write:

$$
\begin{aligned}
I\left(\mathcal{A}_{p}^{3}\right)(z)= & \sum_{(q, s, m) \in \mathcal{A}_{p}^{3}}(q-m)\left(c_{m}^{(p)}\right)^{2}\left(\sum_{\ell=1}^{d_{p}} a_{q \ell}^{(p)} \overline{a_{s \ell}^{(p)}}\right) z^{q+m-1} \bar{z}^{s+m} \\
& -\sum_{s=1}^{\infty} \sum_{m=\delta_{p}^{\prime}+1}^{\infty}(s-m)\left(c_{m}^{(p)}\right)^{2}\left(c_{s}^{(p)}\right)^{2} z^{s+m-1} \bar{z}^{s+m} \\
= & : S_{1}^{\prime}-S_{2}^{\prime} .
\end{aligned}
$$

On the one hand, rather similarly as for (3.64) (observe the precise exponents though),

$$
\left|S_{1}^{\prime}\right| \leq d_{p} \sum_{q=1}^{\infty} q\left(\sup _{1 \leq \ell \leq d_{p}}\left|a_{q \ell}^{(p)}\right|\right)|z|^{q} \sum_{s=1}^{\infty}\left(\sup _{1 \leq \ell \leq d_{p}}\left|a_{s \ell}^{(p)}\right|\right)|z|^{s} \sum_{m=\delta_{p}^{\prime}+1}^{\infty} m\left(c_{m}^{(p)}\right)^{2}|z|^{2 m-1} \text {. }
$$

Again, we deal separately with $\sum_{s=1}^{\delta_{p}^{\prime}}$ and $\sum_{s=\delta_{p}^{\prime}+1}^{+\infty}$ from (3.54), (3.66).

In conclusion, by (2.3), (3.54), (3.65), (3.66), (3.71) and (3.74), we have on $|z| \leq c^{\prime} p^{-A^{\prime}}$,

$$
\left|S_{1}^{\prime}\right| \leq \mathcal{O}\left(p^{4} 2^{-2 \alpha^{\prime} p}\right) \beta_{p}^{\mathbb{D}^{*}}(z)^{2} .
$$

On the other hand, we have by (3.71) on the set $|z| \leq c^{\prime} p^{-A^{\prime}}$,

$$
\begin{aligned}
\left|S_{2}^{\prime}\right| & \leq \sum_{q=1}^{\infty} \sum_{m=\delta_{p}^{\prime}+1}^{\infty}|q-m|\left(c_{m}^{(p)}\right)^{2}\left(c_{q}^{(p)}\right)^{2}|z|^{2 q+2 m-1} \\
& \leq\left(\sum_{q=1}^{\infty} q\left(c_{q}^{(p)}\right)^{2}|z|^{2 q}\right)\left(\sum_{m=\delta_{p}^{\prime}+1}^{\infty} m\left(c_{m}^{(p)}\right)^{2}|z|^{2 m-1}\right) \\
& \leq C\left(\delta_{p}^{\prime} \sum_{q=1}^{\delta_{p}^{\prime}}\left(c_{q}^{(p)}\right)^{2}|z|^{2 q}+p 2^{-2 \alpha^{\prime} p} \beta_{p}^{\mathbb{D}^{*}}(z)\right) p 2^{-2 \alpha^{\prime} p} \beta_{p}^{\mathbb{D}^{*}}(z) \\
& \leq C 2^{-2 \alpha^{\prime} p} p^{2} \beta_{p}^{\mathbb{D}^{*}}(z)^{2} .
\end{aligned}
$$

By (3.73), (3.75) and (3.76) we have on the set $|z| \leq c^{\prime} p^{-A^{\prime}}$,

$$
\left|I\left(\mathcal{A}_{p}^{3}\right)(z)\right|=\mathcal{O}\left(p^{4} 2^{-2 \alpha^{\prime} p}\right) \beta_{p}^{\mathbb{D}^{*}}(z)^{2} .
$$

Conclusion. - We sum up the estimates above (head terms (3.43), (3.44), (3.47), and tail terms (3.62), (3.72), (3.77)) in (3.50), with $\kappa$ any fixed number larger than $\frac{1}{2} \log 2$, and obtain for some $\gamma>0$,

$$
\sup _{|z| \leq c^{\prime} p^{-A^{\prime}}}\left|\frac{d}{d z}\left(\frac{B_{p}}{B_{p}^{\mathbb{D}^{*}}}\right)(z)\right|=\mathcal{O}\left(e^{-\gamma p}\right) .
$$

Applying Theorem 1.1 for $k=1, \delta=0$, we get

$$
\sup _{c^{\prime} p^{-A^{\prime} \leq|z| \leq r}}|z|\left|\log \left(|z|^{2}\right)\right|\left|\frac{d}{d z}\left(B_{p}-B_{p}^{\mathbb{D}^{*}}\right)(z)\right|=\mathcal{O}\left(p^{-\infty}\right),
$$

\footnotetext{
1 Fine uniform control for small indices, rough control via Cauchy formula for large indices, sacrifice of a few powers of $|z|$ and restriction to $|z| \leq c p^{-A}$ for resulting sums.
} 
which can be rephrased as follows:

$$
\sup _{c^{\prime} p^{-A^{\prime} \leq|z| \leq r}}\left|\frac{d}{d z}\left(B_{p}-B_{p}^{\mathbb{D}^{*}}\right)(z)\right|=\mathcal{O}\left(p^{-\infty}\right) .
$$

Estimates (2.8), (3.78), (3.80) yield (1.10) for $k=1$.

Higher $k$-order estimates are established along the same lines: (1) the sum over the set of indices in $\mathcal{A}_{p}^{j}$ where one of indices satisfies $\geq \delta_{p}^{\prime}+1$, will be controlled by a polynomial in $p$ times $2^{-\alpha^{\prime} p} \beta_{p}^{\mathbb{D}^{*}}(z)^{k} ;(2)$ to handle the sum over the set of indices $\leq \delta_{p}^{\prime}$, we observe first that the contribution from the terms with sum of indices $<2 k+2$ is zero, so we will increase $\kappa$ to absorb the exponential factor in the estimates. Thus the analogue of (3.78) holds for $k>1$. We exemplify this for the second derivative $\frac{d^{2}}{d z^{2}}$ to show how the above argument works. From (3.39), we get

$$
\begin{aligned}
& \frac{d^{2}}{d z^{2}} \frac{B_{p}}{B_{p}^{\mathbb{D}^{*}}}(z)=\left(\beta_{p}^{\mathbb{D}^{*}}(z)\right)^{-3} \\
& \quad \times \sum_{q, s, t, m=1}^{\infty}(q-m)(q+m-1-2 t)\left(c_{m}^{(p)}\right)^{2}\left(c_{t}^{(p)}\right)^{2} c_{q}^{(p)} c_{s}^{(p)} \epsilon_{q s} z^{q+m-2+t} \bar{z}^{s+m+t} .
\end{aligned}
$$

It is clear that the contribution of the indices with $q+m+t<5$ is zero, so the trick (3.42) works even in the presence of a $z^{-2}$-term in (3.81).

\section{Applications}

Theorem 1.3 can be interpreted in terms of Kodaira embeddings. Following the seminal papers $[8,11,14,17,23,28,29]$ one of the main applications of the expansion of the Bergman kernel is the convergence of the induced Fubini-Study metrics by Kodaira maps. Let us consider the Kodaira map at level $p \geq 2$ induced by $H_{(2)}^{0}\left(\Sigma, L^{p}\right)$, which is a meromorphic map defined by

$$
J_{p,(2)}: \Sigma \rightarrow \mathbb{P}\left(H_{(2)}^{0}\left(\Sigma, L^{p}\right)^{*}\right) \cong \mathbb{C P}^{d_{p}-1}, x \longmapsto\left\{\sigma \in H_{(2)}^{0}\left(\Sigma, L^{p}\right): \sigma(x)=0\right\}
$$

Recall that by $\left[5\right.$, Remark 3.2] the sections of $H_{(2)}^{0}\left(\Sigma, L^{p}\right)$ extend to holomorphic sections of $L^{p}$ over $\bar{\Sigma}$ that vanish at the punctures and this gives an identification

$$
H_{(2)}^{0}\left(\Sigma, L^{p}\right) \cong\left\{\sigma \in H^{0}\left(\bar{\Sigma}, L^{p}\right):\left.\sigma\right|_{D}=0\right\} .
$$

Let $\sigma_{D}$ be the canonical section of the bundle $\mathscr{O}_{\bar{\Sigma}}(D)$. The map

$$
H^{0}\left(\bar{\Sigma}, L^{p} \otimes \mathscr{O}_{\bar{\Sigma}}(-D)\right) \rightarrow\left\{\sigma \in H^{0}\left(\bar{\Sigma}, L^{p}\right):\left.\sigma\right|_{D}=0\right\}, \quad s \mapsto s \otimes \sigma_{D},
$$

is an isomorphism and we have an identification $H^{0}\left(\bar{\Sigma}, L^{p} \otimes \mathscr{O}_{\bar{\Sigma}}(-D)\right) \otimes \sigma_{D} \cong$ $H_{(2)}^{0}\left(\Sigma, L^{p}\right) \subset H^{0}\left(\bar{\Sigma}, L^{p}\right)$. Since the zero divisor of $\sigma_{D}$ is $D$ we have for $x \in \Sigma$,

$$
\left\{\sigma \in H_{(2)}^{0}\left(\Sigma, L^{p}\right): \sigma(x)=0\right\}=\left\{s \in H^{0}\left(\bar{\Sigma}, L^{p} \otimes \mathscr{O}_{\bar{\Sigma}}(-D)\right): s(x)=0\right\} \otimes \sigma_{D} .
$$


Let $J_{p}$ the Kodaira map defined by $H^{0}\left(\bar{\Sigma}, L^{p} \otimes \mathscr{O}_{\bar{\Sigma}}(-D)\right)$. We have by (4.4) the commutative diagram

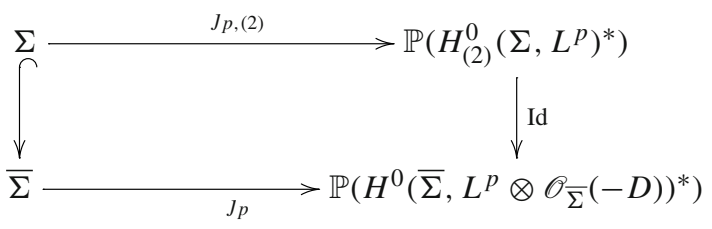

It is well known that $J_{p}$ is a holomorphic embedding for $p$ large enough, namely for all $p$ satisfying $p \operatorname{deg}(L)-N>2 g$ (see [19,p. 215]). Thus $J p$,(2) is also an embedding for $p$ large enough, as the restriction of an embedding of $\bar{\Sigma}$.

The $L^{2}$-metric (2.1) on $H_{(2)}^{0}\left(\Sigma, L^{p}\right)$ induces a Fubini-Study Kähler metric $\omega_{\mathrm{FS}, p}$ on the projective space $\mathbb{P}\left(H_{(2)}^{0}\left(\Sigma, L^{p}\right)^{*}\right)$ and a Fubini-Study Hermitian metric $h_{\mathrm{FS}, p}$ on the hyperplane line bundle $\mathscr{O}(1) \rightarrow \mathbb{P}\left(H_{(2)}^{0}\left(\Sigma, L^{p}\right)^{*}\right)$. By [22,Theorem 5.1.3] $J_{p}$ and $J_{p,(2)}$ induce canonical isomorphisms

$$
J_{p}^{*} \mathscr{O}(1) \simeq L^{p} \otimes \mathscr{O}(-D),\left.\quad j_{p,(2)}^{*} \mathscr{O}(1) \simeq L^{p}\right|_{\Sigma} .
$$

Let $J_{p,(2)}^{*} h_{\mathrm{FS}, p}$ be the Hermitian metric induced by $h_{\mathrm{FS}, p}$ via the isomorphism (4.6) on $\left.L^{p}\right|_{\Sigma}$.

Theorem 4.1 Let $\left(\Sigma, \omega_{\Sigma}, L, h\right)$ fulfill conditions $(\alpha)$ and $(\beta)$. Then as $p \rightarrow \infty$,

$$
\begin{aligned}
& J_{p,(2)}^{*} h_{\mathrm{FS}, p}=\left(1+\mathcal{O}\left(p^{-\infty}\right)\right)\left(B_{p}^{\mathbb{D}^{*}}\right)^{-1} h^{p}, \\
& \frac{1}{p} J_{p,(2)}^{*} \omega_{\mathrm{FS}, p}=\frac{1}{2 \pi} \omega_{\Sigma}+\frac{i}{2 \pi p} \partial \bar{\partial} \log \left(B_{p}^{\mathbb{D}^{*}}\right)+\mathcal{O}\left(p^{-\infty}\right),
\end{aligned}
$$

uniformly on $V_{1} \cup V_{2} \cup \ldots \cup V_{N}$.

Proof We have indeed by [22,Theorem 5.1.3],

$$
J_{p,(2)}^{*} h_{\mathrm{FS}, p}=\left(B_{p}\right)^{-1} h^{p}, \quad \frac{1}{p} J_{p,(2)}^{*} \omega_{\mathrm{FS}, p}=\frac{i}{2 \pi} R^{L}+\frac{i}{2 \pi p} \partial \bar{\partial} \log \left(B_{p}\right),
$$

so (4.7) follows from Theorems 1.2 and 1.3.

We compare next the induced Fubini-Study metrics by $J_{p,(2)}$ on $\Sigma$ and on $\mathbb{D}^{*}$, and show that they differ from each other (modulo the usual identification on $\mathbb{D}_{4 r}^{*}$ in (1.6) with the neighbourhood of a singularity of $\Sigma$ ) by a sequence of $(1,1)$-forms which is $\mathcal{O}\left(p^{-\infty}\right)$ (at every order) with respect to any smooth reference metric on $\mathbb{D}_{r}$ : the situation is just as good as in the smooth setting.

The infinite dimensional projective space $\mathbb{C P}^{\infty}$ is a Hilbert manifold modeled on the space $\ell^{2}$ of square-summable sequences of complex numbers $\left(a_{j}\right)_{j \in \mathbb{N}}$ endowed with the norm $\left\|\left(a_{j}\right)\right\|=\left(\sum_{j \geq 0}\left|a_{j}\right|^{2}\right)^{1 / 2}$. Then $\mathbb{C P}^{\infty}=\ell^{2} \backslash\{0\} / \mathbb{C}^{*}$ and for $a \in \ell^{2}$ we denote by $[a]$ its class in $\mathbb{C P}^{\infty}$. The affine charts are defined as usual by $U_{j}=\left\{[a]: a_{j} \neq 0\right\}$. The Fubini-Study metric $\omega_{\mathrm{FS}, \infty}$ is defined by $\omega_{\mathrm{FS}, \infty}=\frac{i}{2 \pi} \partial \bar{\partial} \log \|a\|^{2}$ to the effect that for a holomorphic map $F: M \rightarrow \mathbb{C P}^{\infty}$ from a complex manifold $M$ to $\mathbb{C P}^{\infty}$ we have $F^{*} \omega_{\mathrm{FS}, \infty}=$ $\frac{i}{2 \pi} \partial \bar{\partial} \log \|F\|^{2}$. We define the Kodaira map of level $p$ associated with $\left(\mathbb{D}^{*}, \omega_{\mathbb{D}} *, \mathbb{C}, h_{\mathbb{D}^{*}}\right)$ by using the orthonormal basis (2.6) of $H_{(2)}^{p}\left(\mathbb{D}^{*}\right)$,

$$
\iota_{p}: \mathbb{D}^{*} \rightarrow \mathbb{C P}^{\infty}, \quad \iota_{p}(z)=\left[c_{1}^{(p)} z, c_{2}^{(p)} z^{2}, \ldots, c_{\ell}^{(p)} z^{\ell}, \ldots\right] \in \mathbb{C P}^{\infty}, \quad z \in \mathbb{D}^{*} .
$$


Theorem 4.2 Suppose that $\mathbb{D}_{4 r}^{*}$ and $V \subset \Sigma$ are identified as in (1.6). On $\mathbb{D}_{4 r}^{*}$ we set

$$
\imath_{p}^{*} \omega_{\mathrm{FS}, \infty}-j_{p,(2)}^{*} \omega_{\mathrm{FS}, p}=\eta_{p} i d z \wedge d \bar{z} .
$$

Then $\eta_{p}$ extends smoothly to $\mathbb{D}_{r}$ and one has, for all $k \geq 0, \ell \geq 0$,

$$
\left\|\eta_{p}\right\|_{C^{k}\left(\mathbb{D}_{r}\right)} \leq C_{k, \ell} p^{-\ell}, \quad \text { as } p \rightarrow \infty,
$$

where $\|\cdot\|_{C^{k}\left(\mathbb{D}_{r}\right)}$ is the usual $C^{k}$-norm on $\mathbb{D}_{r}$.

Proof We first observe that $\iota_{p}$ is an embedding, since already $z \mapsto\left[c_{1}^{(p)} z, c_{2}^{(p)} z^{2}\right] \in \mathbb{C P}^{1}$ is an embedding. We have

$$
\frac{p}{2 \pi} \omega_{\mathbb{D}^{*}}=\iota_{p}^{*} \omega_{\mathrm{FS}, \infty}-\frac{i}{2 \pi} \partial \bar{\partial} \log \left(B_{p}^{\mathbb{D}^{*}}\right)
$$

and consequently on $\mathbb{D}_{r}^{*}$,

$$
\imath_{p}^{*} \omega_{\mathrm{FS}, \infty}-J_{p,(2)}^{*} \omega_{\mathrm{FS}, p}=\frac{i}{2 \pi} \partial \bar{\partial} \log \left(B_{p}^{\mathbb{D}^{*}} / B_{p}\right),
$$

so the assertion follows from Theorem 1.3.

We finish with an application to random Kähler geometry, more precisely to the distribution of zeros of random holomorphic sections $[12,16,26]$.

Let us endow the space $H_{(2)}^{0}\left(\Sigma, L^{p}\right)$ with a Gaussian probability measure $\mu_{p}$ induced by the unitary map $H_{(2)}^{0}\left(\Sigma, L^{p}\right) \cong \mathbb{C}^{d_{p}}$ given by the choice of an orthonormal basis $\left(S_{j}^{p}\right)_{j=1}^{d_{p}}$. Given a section $s \in H_{(2)}^{0}\left(\Sigma, L^{p}\right) \subset H^{0}\left(\bar{\Sigma}, L^{p}\right)$ we denote by $[s=0]$ the zero distribution on $\bar{\Sigma}$ defined by the zero divisor of $s$ on $\bar{\Sigma}$. If the zero divisor of $s$ is given by $\sum m_{j} P_{j}$, where $m_{j} \in \mathbb{N}$ and $P_{j} \in \bar{\Sigma}$, then $[s=0]=\sum m_{j} \delta_{P_{j}}$, where $\delta_{P}$ is the delta distribution at $P \in \bar{\Sigma}$. We denote by $\langle\cdot, \cdot\rangle$ the duality between distributions and test functions. For a test function $\Phi \in C^{\infty}(\bar{\Sigma})$ and $s$ as above we have $\langle[s=0], \Phi\rangle=\sum m_{j} \Phi\left(P_{j}\right)$.

The expectation distribution $\mathbb{E}\left[s_{p}=0\right]$ of the distribution-valued random variable $H_{(2)}^{0}\left(\Sigma, L^{p}\right) \ni s_{p} \mapsto\left[s_{p}=0\right]$ is defined by

$$
\left\langle\mathbb{E}\left[s_{p}=0\right], \Phi\right\rangle=\int_{H_{(2)}^{0}\left(\Sigma, L^{p}\right)}\left\langle\left[s_{p}=0\right], \Phi\right\rangle d \mu_{p}\left(s_{p}\right),
$$

where $\Phi$ is a test function on $\bar{\Sigma}$. We consider the product probability space

$$
(\mathcal{H}, \mu)=\left(\prod_{p=1}^{\infty} H_{(2)}^{0}\left(\Sigma, L^{p}\right), \prod_{p=1}^{\infty} \mu_{p}\right)
$$

Theorem 4.3 (i) The smooth $(1,1)$-form $J_{p,(2)}^{*} \omega_{\mathrm{FS}, p}$ extends to a closed positive $(1,1)$-current on $\bar{\Sigma}$ denoted $\gamma_{p}$ (called Fubini-Study current) and we have $\mathbb{E}\left[s_{p}=0\right]=\gamma_{p}$. (ii) We have $\frac{1}{p} \gamma_{p} \rightarrow \frac{i}{2 \pi} R^{L}$ as $p \rightarrow \infty$, weakly in the sense of currents on $\bar{\Sigma}$, where $R^{L}$ is the curvature current of the singular holomorphic bundle $(L, h)$ on $\bar{\Sigma}$.

(iii) For almost all sequences $\left(s_{p}\right) \in(\mathcal{H}, \mu)$ we have $\frac{1}{p}\left[s_{p}=0\right] \rightarrow \frac{i}{2 \pi} R^{L}$ as $p \rightarrow \infty$, weakly in the sense of currents on $\bar{\Sigma}$. 
Proof The convergence of the Fubini-Study currents $\gamma_{p}$ follows from (4.7). The rest of the assertions follow from the general arguments of [12,Theorems 1.1, 4.3]. The conditions (A)(C) in $[12$, Theorems $1.1,4.3]$ are implied by our hypotheses $(\alpha),(\beta)$ and the required local uniform convergence $\frac{1}{p} \log B_{p} \rightarrow 0$ as $p \rightarrow+\infty$ on $\Sigma$ is a consequence of [22, Theorem 6.1.1].

Acknowledgements We would like to thank Professor Jean-Michel Bismut for helpful discussions. In particular, Theorem 1.3 answers a question raised by him at CIRM in October 2018.

Funding Open Access funding enabled and organized by Projekt DEAL.

Open Access This article is licensed under a Creative Commons Attribution 4.0 International License, which permits use, sharing, adaptation, distribution and reproduction in any medium or format, as long as you give appropriate credit to the original author(s) and the source, provide a link to the Creative Commons licence, and indicate if changes were made. The images or other third party material in this article are included in the article's Creative Commons licence, unless indicated otherwise in a credit line to the material. If material is not included in the article's Creative Commons licence and your intended use is not permitted by statutory regulation or exceeds the permitted use, you will need to obtain permission directly from the copyright holder. To view a copy of this licence, visit http://creativecommons.org/licenses/by/4.0/.

\section{References}

1. Abbes, A., Ullmo, E.: Comparaison des métriques d'Arakelov et de Poincaré sur $X_{0}(N)$. Duke Math. J. 80, 295-307 (1995)

2. Auvray, H.: The space of Poincaré type Kähler metrics. J. Reine Angew. Math. 722, 1-64 (2017)

3. Auvray, H.: Asymptotic properties of extremal Kähler metrics of Poincaré type. Proc. Lond. Math. Soc. (3) 115(4), 813-853 (2017)

4. Auvray, H., Ma, X., Marinescu, G.: Bergman kernels on punctured Riemann surfaces. C. R. Math. Acad. Sci. Paris 354(10), 1018-1022 (2016)

5. Auvray, H., Ma, X., Marinescu, G.: Bergman kernels on punctured Riemann surfaces. Math. Ann. 379(34), 951-1002 (2021)

6. Berman, R., Freixas i Montplet, G.: An arithmetic Hilbert-Samuel theorem for singular Hermitian line bundles and cusp forms. Compos. Math. 150(10), 1703-1728 (2014)

7. Bismut, J.-M. , Lebeau, G.: Complex immersions and Quillen metrics. Inst. Hautes Études Sci. Publ. Math. no. 74 (1991), ii+298 pp. (1992)

8. Bouche, T.: Convergence de la métrique de Fubini-Study d'un fibré linéaire positif. Ann. Inst. Fourier (Grenoble) 40(1), 117-130 (1990)

9. Bruinier, J.H., Burgos Gil, J.I., Kühn, U.: Borcherds products and arithmetic intersection theory on Hilbert modular surfaces. Duke Math. J. 139, 1-88 (2007)

10. Burgos Gil, J.I., Kramer, J., Kühn, U.: Arithmetic characteristic classes of automorphic vector bundles. Doc. Math. 10, 619-716 (2005)

11. Catlin, D.: The Bergman kernel and a theorem of Tian. In: Analysis and Geometry in Several Complex Variables (Katata, 1997), Trends in Mathematics, pp. 1-23. Birkhäuser, Boston (1999)

12. Coman, D., Marinescu, G.: Equidistribution results for singular metrics on line bundles. Ann. Sci. Éc. Norm. Supér. (4) 48(3), 497-536 (2015)

13. Coman, D., Marinescu, G.: On the first order asymptotics of partial Bergman kernels. Ann. Fac. Sci. Éc. Toulouse Math. (6) 26(5), 1193-1210 (2017)

14. Dai, X., Liu, K., Ma, X.: On the asymptotic expansion of Bergman kernel. J. Differ. Geom. 72(1), 1-41 (2006)

15. Dai, X., Liu, K., Ma, X.: A remark on weighted Bergman kernels on orbifolds. Math. Res. Lett. 19(1), 143-148 (2012)

16. Dinh, T.-C., Sibony, N.: Distribution des valeurs de transformations méromorphes et applications. Comment. Math. Helv. 81, 221-258 (2006)

17. Donaldson, S.K.: Scalar curvature and projective embeddings. I. J. Differ. Geom. 59(3), 479-522 (2001)

18. Friedman, J. S., Jorgenson, J., Kramer, J.: Uniform Sup-norm Bounds on Average for Cusp Forms of Higher Weights, Arbeitstagung Bonn 2013, Progress in Mathematics, 319, pp. 127-154. Birkhäuser/Springer (2016) 
19. Griffiths, P., Harris, J.: Principles of Algebraic Geometry. Wiley-Interscience [John Wiley \& Sons], New York (1978)

20. Hsiao, C.-Y.: Projections in several complex variables. Mém. Soc. Math. Fr. (N.S.) No. 123, 131 pp. (2010)

21. Jorgenson, J., Kramer, J.: Bounding the sup-norm of automorphic forms. Geom. Funct. Anal. 14, 12671277 (2004)

22. Ma, X., Marinescu, G.: Holomorphic Morse Inequalities and Bergman Kernels, Progress in Mathematics, 254. Birkhäuser Verlag, Basel (2007)

23. Ma, X., Marinescu, G.: Generalized Bergman kernels on symplectic manifolds. Adv. Math. 217(4), 17561815 (2008)

24. Sun, J.: Estimations of the Bergman kernel of the punctured disc. arXiv: 1706.01018

25. Sun, J., Sun, S.: Projective embedding of log Riemann surfaces and K-stability. J. Geom. Anal. 31(6), 5526-5554 (2021)

26. Shiffman, B., Zelditch, S.: Distribution of zeros of random and quantum chaotic sections of positive line bundles. Commun. Math. Phys. 200, 661-683 (1999)

27. Székelyhidi, G.: Extremal metrics and K-stability (PhD thesis). arXiv:math/0611002

28. Tian, G.: On a set of polarized Kähler metrics on algebraic manifolds. J. Differ. Geom. 32, 99-130 (1990)

29. Zelditch, S.: Szegő kernels and a theorem of Tian. Int. Math. Res. Not. 6, 317-331 (1998)

Publisher's Note Springer Nature remains neutral with regard to jurisdictional claims in published maps and institutional affiliations. 